\title{
Uranium Concentration Monitor Manual 2300 System
}

\author{
LA- $-10350-M$
}

DE85 015359

\author{
D. A. Russo \\ J. K. Sprinkle, Jr. \\ M. M. Stephens
}

\begin{abstract}
DISCLAIMER
This report was prepared as an account of work sponsored by an agency of the United States Goverument. Neither the United States Government nor any agency thereof, nor any of their employees, makes any wurranty, express or implied, or assumes any legal liability or responsibility for the accuracy, completeness, or usefulness of any information, apparatus, product, or process disclosed, or represents that its use would not infringe privately owned rights. Reference herein to any specific commercial product, process, or service by trade name, trademark, manufacturer, or otherwise does not necessarily constitute or imply its endorsement, recommendation, or favoring by the United States Government or any agency thereof. The views and opinions of authors expressed herein do not necessarily state or reflect those of the United States Government or any agency thereof.
\end{abstract}




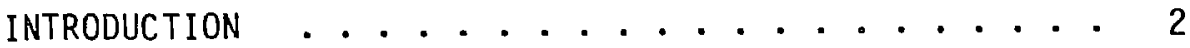

Overview ................ 2

Routine Use .............. 4

EQUIPMENT SUMMARY .................... 5

Detectors and Electronics . . . . . . 5

Adjustment of Analog Electronics . . . . . . 11

Gain.............. . . 11

Stabilization ............... 13

Fabricated Hardware . . . . . . . . . . . . 14

Working Standards and Radioisotopic Sources . . . 16

MICROCOMPUTER HARDWARE . . . . . . . . 17

Front Panel . . . . . . . . . . . 17

PDAC LED Display . . . . . . . . . . 17

Restart Button ............ 17

Keypad .............. 17

Remote AGD Displays ........... 8

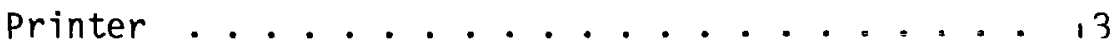

RS-422 Communication.......... 18

Cathode-Ray Tube ............ 19

SOFTWARE OPERATION ................ 20

Use of the Keypad ............. 20

Use of the Menu ............ 20

Use of the CRT ............ . . 33

CRT reatures............. 33

CRT Operations from the Keypad...... 35

Error Messages ............... 37

Error Messages on the PDAC LED Display . . . . 37

Error Messages on the Printout. . . . . . 38 
CALIBRATION AND MEASUREMENT CONTROL . . . . . . 39

The Calibration Curve . . . . . . . . . 39

Normalizing the Calibration ......... 32

Direct Normalization Measurement with Reference

Solution in Standard Cell . . . . . . . . 41

Normalization Measurement by Uranium Foil Assay . . 46

Background Measurement and Aujlistment . . . . . 49

Recommended Measurement Control Procedures . . . . . 51

Weekly Procedure ............. . 51

Monthly Procedure ........... 52

Yearly Procedure . . . . . . . . . 52

Logging Measurement Control Data ...... 53

SOFTWARE SUMMARY ................ 54

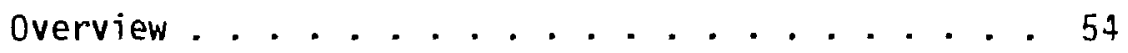

EVALUATION RESULTS ..................... 59

Summary ............... . . 59

REFERENCES ..................... 62

APPENDIX A: CALCULATED 186-keV COUNT RATES VS ELEMENTAL URANIUM CONCENTRATION . . . . . . A.I

APPENDIX B: SOURCE LISTINGS OF DATA ANALYSIS

AND OUTPUT SUBROUTINES . . . . . . . B. 1 
URANIUM CONCENTRATION MONITOR MANUAL 2300 SYSTEM

by

P. A. Russo, J. K. Sprinkie, Jr., and M. M. Stephens

\begin{abstract}
This manual describes the design, operation, and procedures for measurement control for the automated uranium concentration monitor on the 2300 solvent extraction system at the Oak Ridge $Y-12$ Plant. The nonintrusive monitor provides a near-real time readout of uranium concentration at two 1ocations simultaneously in the solvent extraction system for process monitoring and control. Detectors installed at the top of the extraction column and at the bottom of the backwish column acquire spectra of gamma rays from the solvent extraction solutions in the columns. Pulse-height analysis of these spectra gives the concentration of uranium in the organic product of the extraction column and in the aqueous product of the solvent extraction system. The visual readouts of concentrations for p: ocess monitoring are updated every 2 min for both detection systems. Simultaneously, the concentration results are shipped to a remote computer that has been installed by $\mathrm{Y}-12$ to demonstrate automatic control of the solvent extraction system based on input of near-real time process operation information.
\end{abstract}


The uranium concentration monitors for the 2300 solvent extraction system consist of two $\mathrm{NaI}(\mathrm{Tl})$ based detection systems and electronics for automated, continuous assay of uranium concentration monitored nondestructively and nonintrusively at two locations on the $\mathrm{Y}-12$ secondary solvent extraction columns. Two enlarged alphanumeric gas-discharge (AGD) displays give a digital readout of each result of the concentration assay in near-real time for manual process control. The assay results are simultaneously shipped to a remote computer that accepts and interprets realtime operation data and automatically controls the solvent extraction system. Monitor operation and readout are independent of the status of the remote computer, installed initially as part of a demonstration effort by the Y-12 Division of Development.

The 186-keV gamma ray of ${ }^{235} U$ is the signature for the concentration assay. The assay assumes a known ${ }^{235} \mathrm{U}$ enrichment. The two independent pulse-height spectra are acquired and stored simultaneously by multiplexing the two analog signals into one analog-to-digital converter ( $A D C$ ) and then by routing the digitized spectra into separate halves of the multichannei analyzer (MCA) memory. The microprocessor-based MCA automates the data acquisition and readout of results, and provides options for real-time spectral display, generation of a hard copy of the results, and input of data reduction and analysis parameters. 
OVERVIEW

(cont)
An ${ }^{241}$ Am radioisotopic source is attached to each $\mathrm{NaI}(\mathrm{Tl})$ detector in fixed geometry. The net area of the ${ }^{241}$ Am $60-k e V$ photopeak nomalizes the 186-keV photopeak area in each spectrum so that count rate losses do not bias the assay results. The $60-k e V$ photopeak, dominating the count rate in each spectrum, is also used for analog spectrum gain stabilization.

The detection system (through the analog electronics) and the assay method are identical to those of the EUREKA instrument. ${ }^{1-4}$ Refer to the EUREKA Operation Manua ${ }^{5}$ in the appropriate sections below for details of redundant designs.

The computer-based MCA, the PDAC-100, is a Los Alamos prototype instrument. This manual describes PDAC software specific to the 2300 monitor functions and the MCA operation features that are part of the 2300 monitor software package. A separate manua ${ }^{6}$ describes the PDAC-100 hardware and operating system.

The monitor operates unatiended in the continuous assay mode $(120 \mathrm{~s} / \mathrm{cycle})$ on the organic product of the extraction column (detector $A$ ) and on the aqueous product of the solvent extraction system (detector B). Relocation of the monitor detectors to other points on the solvent extraction system is possible with appropriate changes in the concentration assay parameters. 
ROITINE USE

The process operator uses the monitors by visuai observation of the uranium concentration on the two AGD displays. These displays, labeled "2310 ORGANIC" and "2314 AQUEOUS," are on the third floor adjacent to the operations logging area between the 1300 and 2300 solvent extraction systems. The readout of the corresponding uranium concentration (left side of each AGD display), in grams per liter to the nearest tenth, is updated once every 2 min for the normal assay cycle. The right side of each AGD display gives the time remaining, to the nearest second, for the present cycle.

Use of monitors for automatic control of the solvent extraction system requires reading specific information from an alphanumeric data file. At the end of each assay cycle, the monitor ships the file by RS-422 communications to a remote computer responsible for automation of the control. 
DETECTORS AND ELECTRONICS
The NaI(Tl) detectors are 3.2-cm-diam by $1.3-\mathrm{cm}-$ thick crystals integrally mounted to 12-pin, 3.8-cm-diam photomultiplier tubes. The tube bases contain preamplifiers, whose power is tapped from the high-voltage input to the base. The preamplifier signals are amplified and shaped by linear amplifiers that use an internal singlechannel analyzer for analog gain stabilization. The amplifier outputs are multiplexed into a single $A, D C$, and the digitized spectra are stored in separate halves of the 1024-channe1 MCA memory. A riard-copy unit prints both assay results, reduced data, and the assay parameters. Two remote digital readouts display the concentration result from each assay in near-real time. RS-422 communications lines ship the assay results (the complete print file) to a remote computer.

Table I lists the electronics components. Figure 1 is a block diagram of the electronics and detectors. Table II gives the initial electronics settings. Figure 2 is a photograph of the alectronics inside the environmentally controlled cabinet adjacent to the solvent extraction system. Instrunient air continuously feeds the environmental cabinet.

Figure 3 shows the hardware modifications to the PDAC-100 required to route two spectra into separate halves of the analyzer memory. Figure 4 shows the wiring diagrams for the remote displays, printer, and RS-422 communications. 
TABLE I

ELECTRONICS COMPONENTS

\begin{tabular}{|c|c|c|c|}
\hline Model No. & Component & Manufacturer & $\begin{array}{l}\text { No. of } \\
\text { Units }\end{array}$ \\
\hline $1.25 M .50 / 1.5$ & $\mathrm{NaI}(T I)$ detector & Bicron & 2 \\
\hline$P A-12-x$ & Preamplifier base & Bicron & 2 \\
\hline$N A-25$ & High voltage supply & Harshaw & 1 \\
\hline $4001 \mathrm{C} / 4020$ & NIM power bin & EG\&G-ortec & 1 \\
\hline$N A-23$ & Stabilized amplifier & Harshaw & 2 \\
\hline 8222 & Multiplexer-router & Canberra & 1 \\
\hline 3075 & $A D C$ & Canberra & 1 \\
\hline PDAC-100 & MCA & $\begin{array}{l}\text { Prototype (Los Alanos } \\
\text { National Laboratory) }\end{array}$ & 1 \\
\hline APP -48 & Printer & Datel-Intersil & 1 \\
\hline$h-416$ & LED display & Cherry & 2 \\
\hline
\end{tabular}

TABLE II

INITIAL ELECTRONICS SETTINGS

$\frac{2 \text { NA-23 Stabilized Amplifier }}{\text { Pularity }}$
$\begin{array}{ll}\text { Output } & \text { Bipolar } \\ \text { Modes } & \text { Track, Differential }\end{array}$

Detector $\quad C G \quad F G \quad E \quad \underline{F E}$

$\begin{array}{lllll}\text { A } & 40 & 0.90 & 72 & 70\end{array}$

$\begin{array}{lllll}\text { B } & 160 & 0.55 & 67 & 70\end{array}$

\begin{tabular}{cllll} 
High Voltage Supply & \multicolumn{2}{c}{ ADC } & & Multiplexer-Router \\
\cline { 2 - 2 } $650 \mathrm{~V}$ & Kanye $1 \mathrm{k}$ & & Module & 1 \\
& Gain 512 & Memory & $1 \mathrm{k}$ \\
& OFF 0 & Expander & OFF \\
& LLO 11 & Inputs & 2 \\
& Modes & PHA & & \\
& & ANTI & &
\end{tabular}




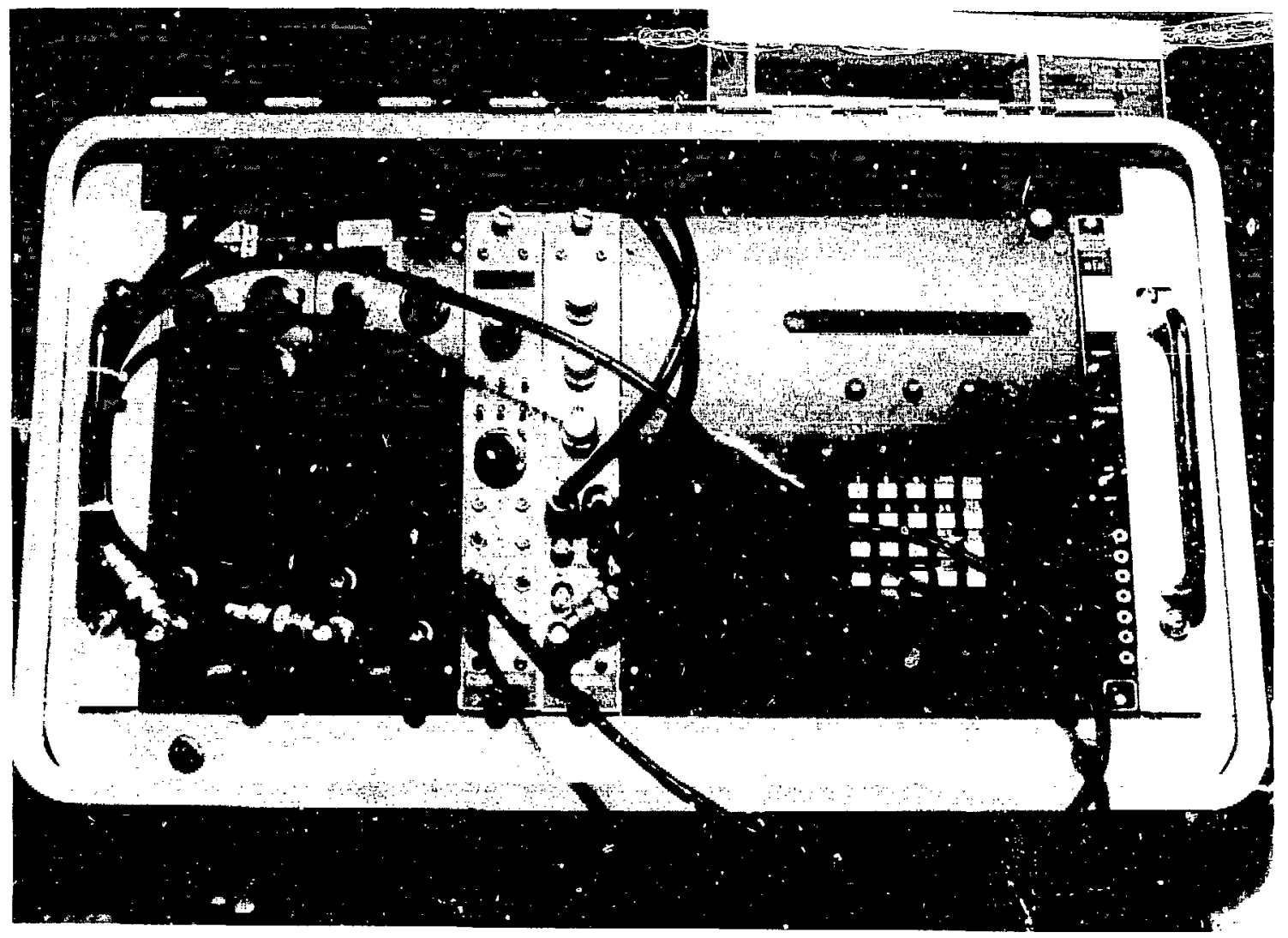

Fig. 2. Photograph of the electronics inside the environmental enclosure (with front Plexiglas cover removed). 


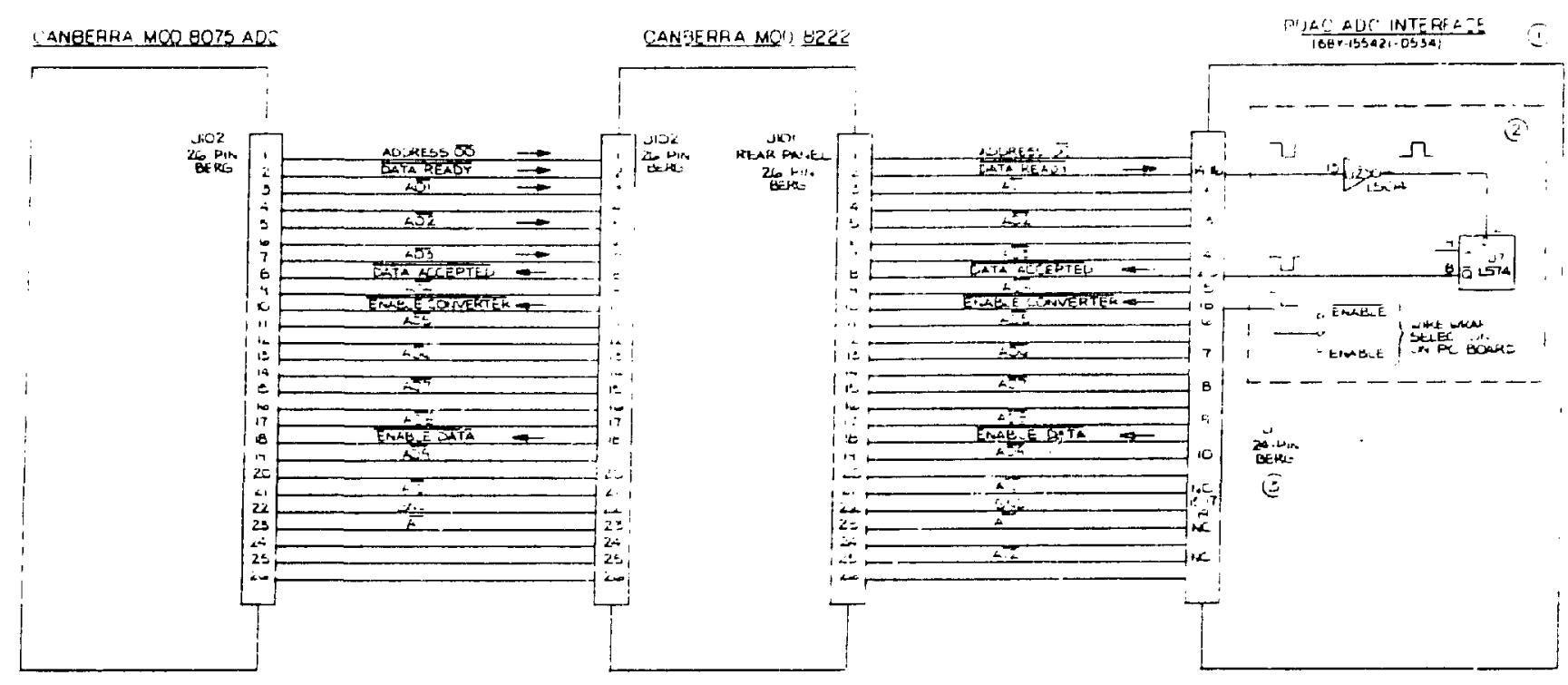

Fig. 3. I1lustration of the interconnections between the PDAC-100 MCA, the 8075 ADC, and the 8222 mixer-router. 


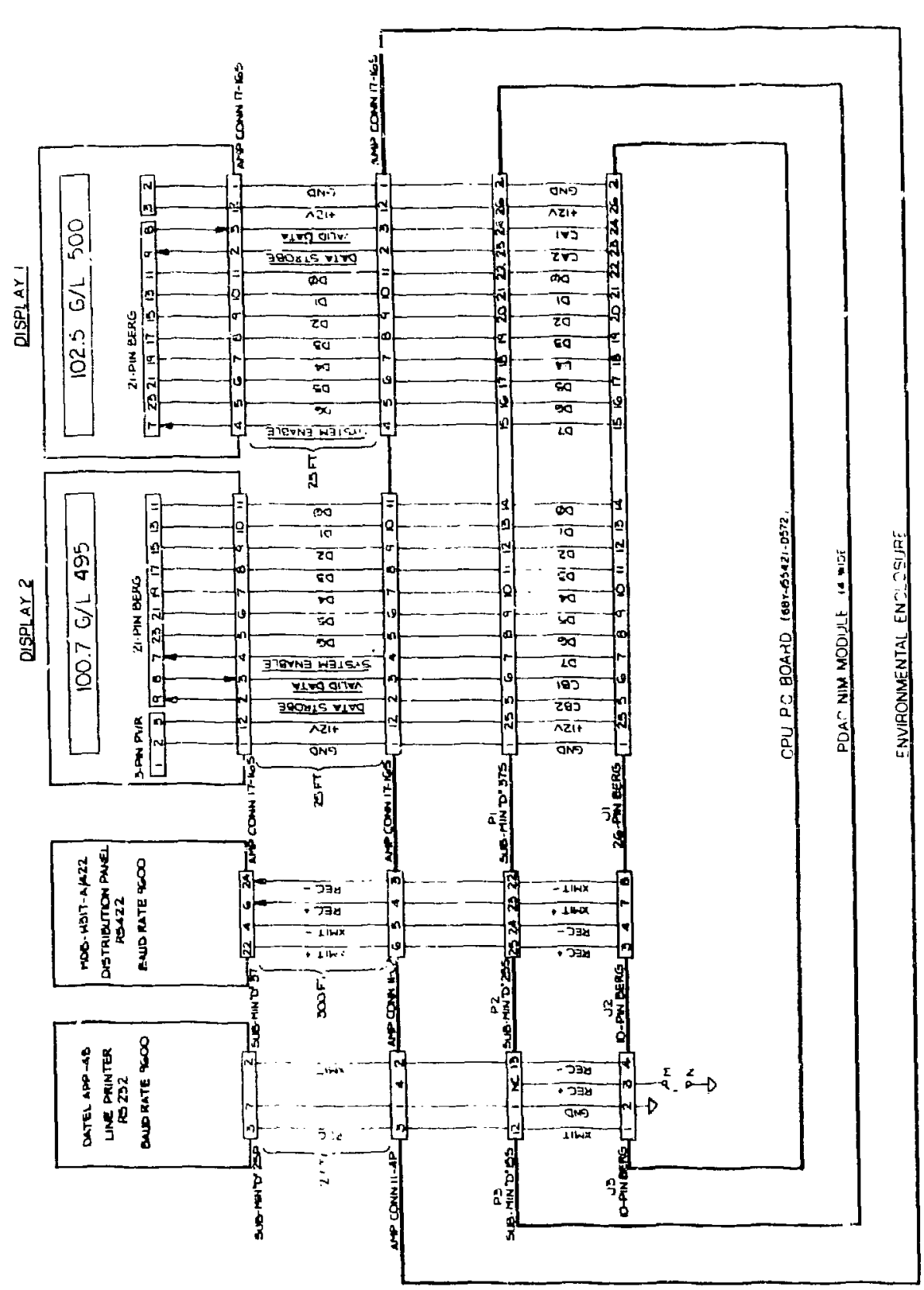

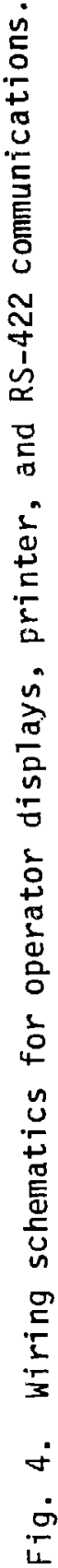




\section{ADJUSTMENT OF ANALOG ELECTRONICS}

Replacement of a detector or amplifier or the nonroutine occurrence of a large change in the electronic gain of an existing detection system requires readjustmait of the analog electronics.

Under normal circumstances, the NA-23 stabilized amplifier should adequately compensate for changes in gain in the detectors or in the amplifier itself. However, if drifts become large enough to exceed the tracking ranje of the NA-23, some adjustment of amplifier gain might be necessary. Adjust the gain if ore of the two red lights (indicating that the $\mathrm{NA}-23$ tracking range is exceeded) fiickers on and uff or stays on, or if a new detector or a new NA-23 amplifier is installed. Minimize the airflow into the environmental cabinet by closing the Plexigias cover immediately after each adjustment because a change of temperature in the electronics will also shift the gain.

Use the following procedure to adjust the gain:

(1) Switch the NA-23 TRACK/DISABLE switch to DISABLE.

(2) Irsert the uranium foil into the detector holder and, with the detector high voltage on, examine the linear output of the NA-23 with an oscilloscope. The 60-keV photopeak puise height should be approximately $1.75 \mathrm{~V}$. 


\section{ADJUSTMENT OF ANALOG \\ ELECTRONICS}

Gain

(cont)
(3) Record the original amplifier fine gain setting. Then, if necessary, carefully adjust the amplifier fine gain to achieve the desired 1.75-V pulse height.

(4) Switch the NA-23 TRACK/DISABI.E switch to TRACK.

(5) Carefully fine tune the amplifier fine gain to achieve an alternate flickering of the two green lights indicating that tracking operation is mid-range. Push the RESET button on the NA-23 after each adjustment of the fine gain.

(6) Verify the proper gain setting by performing a foil assay. The $60-k e V$ peak centroid should be in the prescribed channel $1100.0 \pm 0.5$ for detector $A$ and $612 \pm 0.5$ for detector $B$ ). Check the foil $\mathrm{P}_{2}$ ' value to verify a constant normalization. (Refer to NORMALIZATION MEASUREMENT BY URANIIMM FOIL ASSAY, pp. 46-49 under CALIBRATION AND MEASUREMENT CONTROL.)

(7) Record the new amplifier fine gain setting in the monitor log book. (Refer to Logging Measurement Control Data, p. 53 under CALIBRATION AND MEASUREMENT CONTROL.) 


\section{ADJUSTMENT OF ANALOG}

ELECTRONICS

(cont)

Stabilization

Drifts may occur with time in the stabilizer threshold (adjustable with the "E" control potentiometer of the NA-23 amplifier). The result is a gradual shift in the measured centroid of the $60-\mathrm{keV}$ photopeak. The centroid channel should be $100 \pm 2$ for detector $A$ and $612 \pm 2$ for detector B. The monitor code flags shifts outside these limits. Under no circumstances should frequent (that is, monthly) adjustments for these effects be required.

When an adjustment is required [that is, when CENT(DEV), the deviation in the $60-\mathrm{keV}$ centroid channei, reaches or exceeds \pm 2 for either detector], the following procedures are recommended:

(1) Connect and power up the printer.

(2) Note the measured CENT (DEV) in number of channels for the $60-\mathrm{keV}$ photopeak of the detector that requires adjustment. Compute the approximate magnitude of the adjustment using the formula

Adjustment (turns) $=[$ CENT(DEV)/100] $E$ (turns) where $E$ is the "E" control potentiometer setting. The required adjustment is a very small fraction of a full turn. 


\section{ADJUSTMENT OF ANALOG \\ ELECTRONICS}

Stabilization

(cont)
(3) Open the front panel of the electronics enclosure. Adjust "E." Close the Plexiglas panel immediately after making the adjustment.

(4) With the detector mounted on the column and the uranium foil inserted in the slot of the detector shield, do a foil nieasurement and obser e the $60-\mathrm{keV}$ centroid listed in the printout.

(5) Continue checking the $60-\mathrm{keV}$ centroid to determine if (or how much) additional "E" adjustment is required and repeat steps (3) and (4) if necessary.

(6) After the adjustment of "E" has established the required centroid for the 60-kev peak, repeat the foil assay. In the final printout, verify that the $60-k e v$ centroid is within a few tenths of the desired channel. Then check the foil P2' value to verify a constant normalization.

\section{FABRICATED HARDWARE}

The fabricated hardware consists of stainlesssteel-encased lead shields for the gamma-ray detectors, and stainless steel holders and brackets for mounting the detectors onto or adjacent to the columns.

The holder and bracket for detector $A$ are affixed to external support structure so that the detector 
FABRICATED HARDWARE (cont) views (through 'glass) the pure organic solution on the phase separator of the extraction column of the secondary solvent extraction system.

The holder and pracket for detector B mount directly on the backwash coiumn of the secondary solvent extraction system so that the detector views (through stainless ateel) the pure aqueous product solution in the inactive region at the bottom of the column.

Mechanical design drawings $68 Y-155450, D-1$ through D-4, specify the detector shields and holderbracket hardware. Copies of these drawings appear in Appendix B, pages B1 through B4 of the EUREKA Operation Manual. 5 Figure 5 shows detector $B$ inside the lead shield mounted at the bottom of the 2300 backwash column for assay of the aqueous product solution.

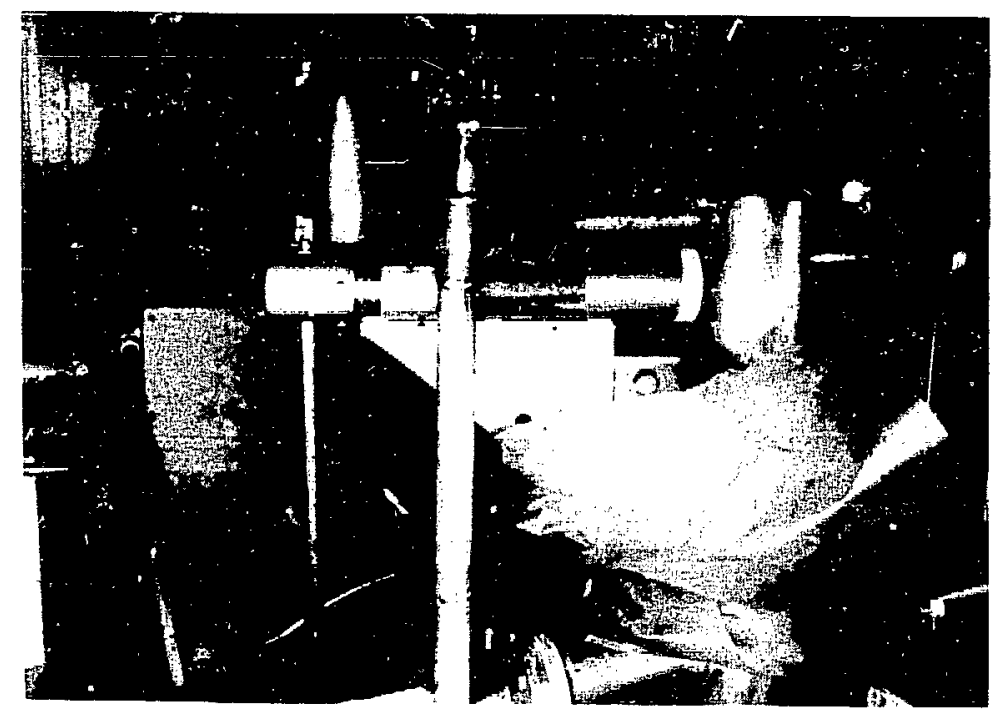

Fig. 5. Detector B molinted at the bottom of the 2300 system backwash column. 
WORKING STANDARDS AND RADIOISOTOPIC SOURCES
Each NaI(TI) detector is mounted in its shield with a 50-nCi ${ }^{241}$ Am radioisotopic source lodtained from Isotope Products Laboratories, Type $M$, grade $C$ ) attached to the front of the detector in fixed geometry with respect to the crystal. The 60-keV gamma ray emitted by ${ }^{241} \mathrm{Am}$ is used to correct for count rate losses. The 60-keV gamma ray is also used for analog gain stabilization.

Each $\mathrm{NaI}(T \mathrm{~T})$ detector is equipped with an enriched uranium mietal foil (working standard) mounted in a tungsten hoider. The foils are $1.3 \mathrm{~cm}$ in diameter and $0.13 \mathrm{~cm}$ thick, and each contains $3 \mathrm{~g}$ of 97\%-enriched uranium mietal. The working standards are used to check the normalization of each detector calibration. Each tungsten holder mounts in a slot in the collimator portion of the detector shield, the uranium foil positioned approximately $5 \mathrm{~cm}$ in front of the crystal. The two foils are not identical; consequently, they cannot be interchanged. 
FRONT PANEL

PDAC LED Display

Restart Button

Keypad
The front panel of the PDAC consists of the lightemitting diode (LED) display, the restart button ! second of three buttons in a row above the keypad), and the keypad. Refer to Fig. '2.

The 16-character LED display normally shows the uranium concentration computed from spectrum $A$ at the end of the most recently completed cycle and the elapsed time for the current cycle. When the LOOK key is pushed, the display sequentially shows the date and time. If the user terminates the normal (assay) operation, then the LED displays menu options and prompts user input.

At power up, the program initializes all variables and starts the first assay cycle automatically. The restart button perfortis the same functions as a power up except that it does not clear the random-access memory (RAM). It can be used as a fast method of starting fresh with cycle 1. In this manual, the word "restart" refers to either a power up or the use of the restart button.

The two unlabeled buttons on either side of the restart button and the five buttons to the left of the keypad have no function.

The 20-key keypad is the only input device on the monitor. Each key is labeled. Some keys have a dual purpose, one for numeric input in the menu mode and one for cathode-ray tube (CRT) operations during data acquisition. For example, the same 
FRONT PANEL

Keypad

(cont)

key is isea ror the decimal point and for changing che vertical scale (YSCL). To use the PDAC keypad, remove the Plexiglas panel on the front of the environmental cabinet. This is not intended auring routine monitoring operations. Restrict the keypad use to needs associated with instrument setup and diagnostics.

REMOTE AGD DISPLAYS

PRINTER

RS-422 COMMUNICATION

As another option, a 9600-baud RS-422 interface
can communicate the monitor assay results to the
PDP 11-23 computer. The output file is identical

As another option, a 9600 -baud RS-422 interface
can communicate the monitor assay results to the
PDP $11-23$ computer. The output file is identical

As another option, a 9600 -baud RS-422 interface
can communicate the monitor assay results to the
PDP $11-23$ computer. The output file is identical to that which is printed.

The two remote 16-character enlarged AGD displays show the uranium concentration computed from spectrum $A$ or $B$ and the elapsed time for the current cycle. Each display is dedicated to one of the two monitor detectors.

The optional 48-character printer logs information about each assay cycle: date and time, regions-of-interest (ROIs) and associated uncertainties, reduced data, and concentration results. It also prints the values of system parameters whenever they are changed. Keep the printer covered and unplugged from the electronics bin and the ac power whe i not in use. to that which is printed. 
CATHODE-RAY TUBE

The optional CRT displays the gamma-ray spectra. It contains a 1024-channel display with intensified ROIs and alphanumerics showing display parameters. Use of the CRT requires connecting the $X$, $Y$, and $Z$ connectors on the side of the environmental cabinet to the CRT display and powering up the display unit. 
USE OF THE KEYPAD

STOP

LOOK
Figure 6 shoiss an enlarged view of the keypad. During data acquisition, only the STOP and LOOK keys function. It the CRT is in use, the keys controlling the CRT also function. The only key recognized during the last $15 \mathrm{~s}$ of a cycle is STOP. The remaining keys are used in dialogues initiated by the menu.

Use the STOP key to stop data acquisition. Confirm by answering YES to the question STOP ACQ? The menu then appearj. In CRT wait mode, use the STOP key to end the pause after acquisition. Coniirm by answering YES to the question STOP WAITINE?. To activate the STOP while computation or printing are in progress (LED display reads "BUSY"), press STOP during the compute-print sequence after printuut of ROIs.

During data acquisition, use the LOOK key to view the current date and time, which appear on the LED dispiay for $3 \mathrm{~s}$. Other information displayed following the date and time display is not applicable to the 2300 monitor.

The menu is a set of questions (requiring a YES or No response) providing choices on tasks. The answer NO causes the next menu question to appear. The answer YES causes the task to begin. When a task is finished, the menu appears from the top again. 


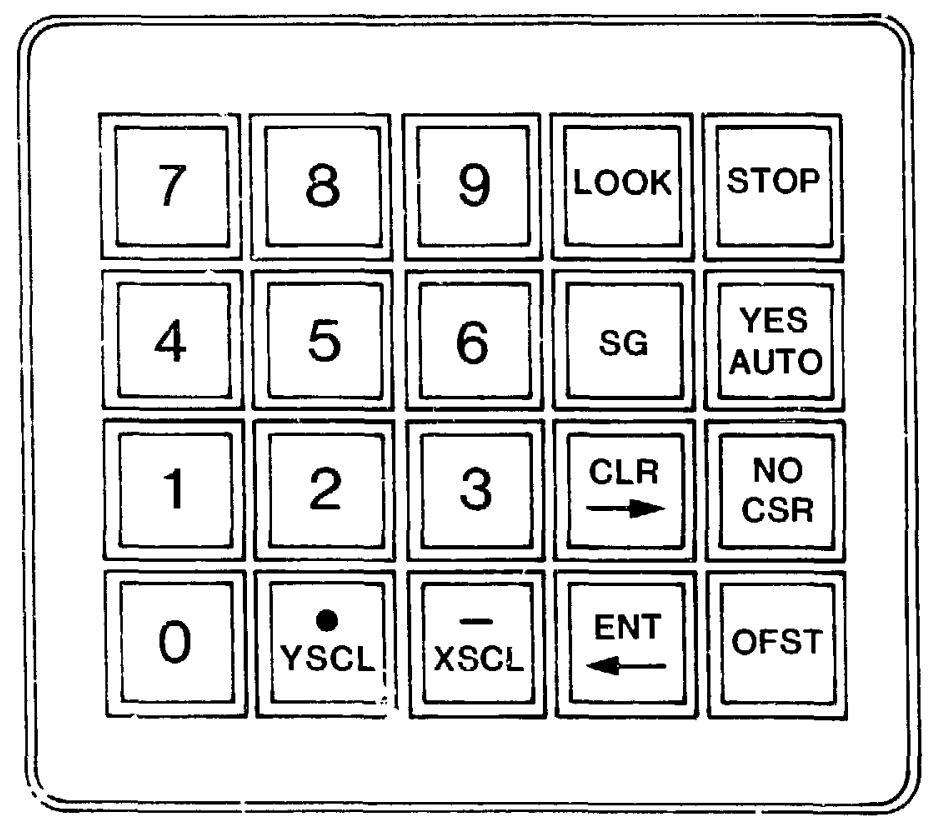

Fig. 6. The PDAC keypad. 
USE OF THE MENU

(cont)
Most of the keys on the PDAC keypad function in dialogues initiated by the menu. The following input converitions appiy to use of the menu:

- When the LED display flashes, the program is waiting for a keypad response.

- When a question mark (?) appears (as in the menu choices!, answer the question YES or NO; no other response is accepted.

- When a right bracket ( $>$ ) appears, a numerical value is expected. To enter a numerical value, use the 0-9, decimal point loptional with integers), and minus keys. The digits appear on the LED display as you push the keys. To complete the entry, push ENT; the LED display is then cleared. After a mistake, push CLR and start ave'n,

Questions appear in the menu in the following order:

START ASSAY?

Answer YES for continuous assay cycles, the normal mode of operation enabled automatically at restart. Cycling continues until the STOP key is pushed. The most recent concentration result and the elapsed time for the current cycle appear on the LED display during acquisition. At ihe end of each cycle, the data are analyzed, and a new data acquisition cycle commences simultariecusly. Output (to LEDs, printer, and PDP 11-23, depending on device selection) follows the data analysis. Figure 7 illustrates the output file. 


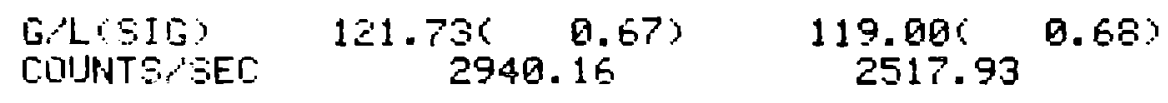

\begin{tabular}{|c|c|c|}
\hline $\begin{array}{l}\text { REGLTN } \\
\text { F1. MEAN }\end{array}$ & $\left.100.580^{7.2051 \%} \because 00\right)$ & $612.711^{6.9189 \%}$ \\
\hline
\end{tabular}

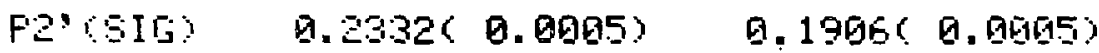

F1'(SIG) 945494.( 1129.) 962905.( 1115.)

1 BALKGFOUNA REGIOHSS) FOR 1 SE KEY FEAK (HB)

Fe(sIG) 221958.96( 487.56) 1656136.96( 412.4Q)

F1(SIG) 939166.25(1121.84) 857361.75(1105.23)

\begin{tabular}{|c|c|}
\hline $\begin{array}{l}\text { H1SE } \\
\text { P18E } \\
\text { L186 } \\
\text { HEEG } \\
\text { FHEG } \\
\text { LEEG }\end{array}$ & $\begin{array}{r}\text { BETECTOR A } \\
8179 . \\
229538 . \\
8179 . \\
799 . \\
994287 . \\
3472 .\end{array}$ \\
\hline
\end{tabular}

6G9 SETIND RINN ENLING 12/15:33 11:96
GLCSIG)
COURTSGEE
124.120 .9 .69 2985.50
118.764 ‥6i\% 2558.56

RESLTN

F'1 MEAN

$$
\text { 7.2159\% }
$$

190.506

0. 09.)

$6.9418 \%$

Fe?

$0.2352($ Q. 0665 ) E.12.77? Q.

F1'SIG 957721.6 1140.5

Q. 1904 ( Q. 010.5$)$ 876951.(1123.)

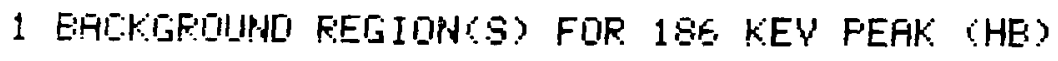

F20IG) 226204.96( 492.66) 167540.950 415.74)

F16IG) 951461.71(1132.67) 871164.76(1116.20)

\begin{tabular}{|c|c|c|}
\hline $\begin{array}{l}\text { H18E } \\
\text { P1EE } \\
\text { L1Es } \\
\text { HBEQ } \\
\text { F'E }\end{array}$ & $\begin{array}{r}\text { LIETECTOR A } \\
8256 . \\
234461 . \\
8256 . \\
8324 . \\
1019564 . \\
3595 .\end{array}$ & $\begin{array}{r}\text { CIETELTUK B } \\
2650 . \\
179191 . \\
2650 . \\
4691 . \\
944641 . \\
13230 .\end{array}$ \\
\hline
\end{tabular}

EĞ GELOND RUN ENINIS $12,15 / 83$ 19:56

Fig. 7. Sample printout of the results from two 600-s assay cycles. 
USE OF THE MENU

(cont)

CAL IBRATE?

Answer YES for continuous assay cycles. (The operation is identical to that following a YES response to "START ASSAY?")

DATE/TIME?

Answer YES to verify or change the date and time. If either is incorrect, answer NO to the question $0 K$ ?, and enter the current month, day, year, hour, and minute (24-h clock) as promoted.

CHANGE DEVICES?

Answer YES to select which devices will be used with the system.

CRT?

Answer YES for a display of the spectrum on the optional CRT. Answer NO for nomal operation. Answer NO if the CRT is not in use.

WAIT MODE?

Wait mode causes a pause after every cycle for viewing the spectrum. End the pause with the STOP key. This question appears only if the CRT is selected. Answer NO for nomal operation.

LP232?

Answer YES to select the local printer to print results. Answer NO otherwise. Answer YES for normal operation. 


\section{USE OF THE MENU}

CHANGE DEVICES? (cont)
LP422?

Answer YES to transmit results to the PDP 11-23. Answer NO otherwise. Answer YES for normal operation.

DUMP SPECTRUM?

Answer YES to print the 1024-channel spectrum on the line printer after each assay. Answer NO otherwise. Answer NO for nomal operation.

REMOTE PANEL?

Answer NO (not applicable).

STRIP CHART?

Answer NO (not applicable).

RENIOTE LEDS?

Answer YES to display the most recent concentration results on the remote AGDs. Answer No otherwise. Answer YES for normal operation.

The device selections are then written to electrically alterable read-only memory (EAROM), where they are stored for the next restart.

CHANGE PARAMS?

Answer YES to view or change system parameters. Parameters appear in three groups. The first contains the miscellaneous constants. The other groups are parameters for measurement control and calibration constants; any group can be bypassed. Refer to Fig. 8 for a line printer listing of the typical parameters. 


\begin{tabular}{|c|c|c|c|c|c|c|}
\hline $\begin{array}{ll}F & 9 \\
F & 8 \\
F & 7 \\
F & 5 \\
P & 5 \\
F & 4 \\
F & 5 \\
F & 2 \\
F & 1 \\
F & 1\end{array}$ & $\begin{array}{l}= \\
= \\
= \\
= \\
= \\
= \\
= \\
= \\
= \\
=\end{array}$ & \multicolumn{3}{|c|}{$\begin{array}{r}0.751963 E-01 \\
-0.155333 E+01 \\
0.136631 E+02 \\
-0.654702 E+02 \\
0.1950191 E+03 \\
-0.353544 E+03 \\
0.3896 .66 E+03 \\
-0.244013 E+03 \\
0.967819 E+02 \\
-0.938272 E+01 \\
\text { A }\end{array}$} & \multicolumn{2}{|c|}{$\begin{array}{r}0.555221 E-01 \\
-0.111983 E+01 \\
0.945527 E+01 \\
-0.444919 E+02 \\
0.125186 E+03 \\
-0.220558 E+03 \\
0.234045 E+03 \\
-0.139391 E+03 \\
0.627836 E+02 \\
-0.484948 E+01\end{array}$} \\
\hline & FIIER & FDL'Y & $\mathrm{E}=$ & 9 & NDENA = & $0.176600 \mathrm{E}+02$ \\
\hline & F:IIEF: & FDLY & $\mathrm{A}=$ & 9 & NDFPA = & $99 E+9$ \\
\hline
\end{tabular}

\begin{tabular}{|c|c|c|}
\hline $\begin{array}{l}\text { CILTH = } \\
\text { MEAN = } \\
\text { PESL = } \\
\text { CIF-2 }\end{array}$ & 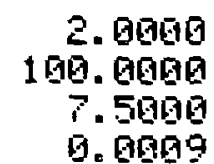 & $\begin{array}{r}\text { 2. } 3090 \\
611.9999 \\
7.5610 \\
9.9096\end{array}$ \\
\hline
\end{tabular}

$\mathrm{IF}-\mathrm{Z}=$ 1. $1 \mathrm{BgG} \quad \mathrm{HLFY}=431.9999$

GEEINALIS FER CUCLE = 6R日

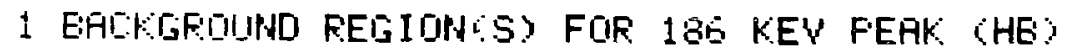

\begin{tabular}{|c|c|c|c|}
\hline 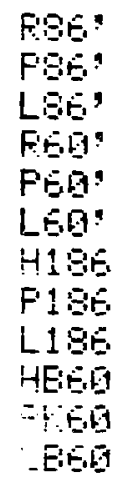 & $\begin{array}{l}912 \\
762 \\
912 \\
630 \\
589 \\
582 \\
499 \\
2519 \\
491 \\
121 \\
72 \\
66\end{array}$ & $\begin{array}{l}- \\
- \\
- \\
- \\
- \\
- \\
- \\
- \\
- \\
- \\
- \\
-\end{array}$ & $\begin{array}{r}1992 \\
852 \\
1092 \\
634 \\
638 \\
586 \\
490 \\
349 \\
490 \\
125 \\
119 \\
78\end{array}$ \\
\hline
\end{tabular}

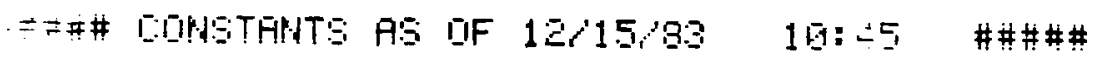

Fig. 8. Sample printout of the parameter file. 
USE OF THE MENU

CHANGE PARAMS?

(cont)
DEV/SG LIMITS?

Answer NO (not applicable).

MISC CONSTANTS?

Answer YES to change or view.

$\mathrm{SEC} / \mathrm{CY}=$

Seconds per cycle (clock time).

$S G=$

Not applicable; enter displayed value.

$C P-2=$

Constant multiply:g 186-keV background ROI total before subtraction from peak total.

$D P-2=$

Background 186/60 subtracted from measured ratio for detector A 'typically less than $0.001)$.

NOW B

$\mathrm{DP}-2=$

Background 186/60 subtracted from measured ratio for detector $B$ (typically less than $0.001)$.

HLFY $=$

Half-life, in years, of normalization source (432.0 for ${ }^{241} \mathrm{Am}$ ). 
USE OF THE MENU

CHANGE PARAMS?

(cont)
MEAS CONTROL?

Answer YES to change or view.

RESL =

Allowed 60-keV photopeak resolution for detector A (per cent). Typical value is $7 \%$.

MEAN $=$

Expected centroid of the $60-\mathrm{keV}$ photopeak for detector A (channel). Typical value is 100 .

DELTA =

Allowed delta around the centroid of the 60-keV photopeak for detector A (channels). Typical value is 2 .

NOW B

RESL $=$

Allowed 60-keV photopeak resolution for detector B (per cent). Typical value is $7 \%$.

MEAN =

Expected centroid of the 60-keV photopeak for detector B (channel). Typical value is 612 .

DELTA =

Allowed delta around the centroid of the 60-keV photopeak for detector B (channels). Typical value is 2 . 
USE OF THE MENU

CHANGE PARAMS?

(cont)
CAL IBRATION?

Answer YES tn change or view.

POLY A FIRST

ORDR $P=$

Order of polynomial fit to calibration curve for detector A. Typical value is 9.

NORM $=$

Normalization factor for detector A counting efficiency. Typical values range from 15 to 20.

$\mathrm{PO}=$

Zero-order coefficient of polynomial fit to calibration curve for detector $A$.

$\mathrm{P1}=$

First-order coefficient of polynomial fit to calibration curve for detector $A$.

$P($ ORDR $P)=$

(ORDR P)-order coefficient of polynomial fit to calibration curve for detector $A$.

NOW B

ORDR $\mathrm{P}=$

(Analogous to ORDR $P$ for POLY A). 
USE OF THE MENU

CHANGE PARAMS?
CALIBRATION? (cont)

NORM $=$

(Analogous to NORM FOR POLY A).

PO $=$

(Analogous to PO FOR POLY A).

$\mathrm{P} 1=$

(Ana logous to P1 for POLY A).

:

$\mathrm{P}($ ORDR $\mathrm{P})=$

[Analogous to $P(O R D R P)$ for POLY $A$ ].

To change a parameter, enter its new value on the keypad and push ENT. To retain the displayed value, push ENT. The new parameter file is printed out and written to EAROM. At this point, the menu will again display "START ASSAY?"

CHANGE ROIS?

Answer YES to view or change ROI channels. Input the left and then right channel for each ROI as follows:

$$
\begin{aligned}
L B 60 \mathrm{~L}= & \text { Low background, } 60 \mathrm{keV}, \text { detector } A, \\
& \text { left channel. }
\end{aligned}
$$


USE OF THE MENU

CHANGE ROIS?

(cont)
LB60 R = Low background, $60 \mathrm{keV}$, detector $A$, right channel.

PK60 L = Peak, $60 \mathrm{keV}$, detector A, left channel.

PK60 R = Peak, 6C keV, detector $A$, right channel.

HB60 L = High background, $60 \mathrm{keV}$, detector $A$, left channel.

HB60 R = High background, $60 \mathrm{keV}$, detector $A$, right channel.

L186 L = Low background, $186 \mathrm{keV}$, detector A, left channel.

L186 R = Low background, $186 \mathrm{keV}$, detector A, right channel.

P186 L = Peak, $186 \mathrm{keV}$, detector A, left channe 1 .

P186 R = Peak, $186 \mathrm{keV}$, detector A, right channe1.

H186 L = High background, $186 \mathrm{keV}$, detector A, left channel.

H186 R = High background, $186 \mathrm{keV}$, detector A, right channel. 
USE OF THE MENU

CHANGE ROIS?

(cont)
${\mathrm{L} 60^{\prime} \mathrm{L}}^{\prime}=$ Low background, $60 \mathrm{keV}$, detector $\mathrm{B}$, left channel.

$L 60^{\prime} \mathrm{R}=$ Low background, $60 \mathrm{keV}$, detector $B$, right channel.

P60' L = Peak, $60 \mathrm{keV}$, detector B, left channel.

$P 60^{\prime} \mathrm{R}=$ Peak, $60 \mathrm{keV}$, detector $\mathrm{B}$, right channel.

R60' $L=$ High background, $60 \mathrm{keV}$, detector $B$, left channel.

R60' $\mathrm{R}=$ High background, $60 \mathrm{keV}$, detector $\mathrm{B}$, right channel.

L86' L = Low background, $186 \mathrm{keV}$, detector $B$, left channel.

L86' $\mathrm{R}=$ Low background, $186 \mathrm{keV}$, detector B, right channel.

$\begin{aligned} \text { P86' } \mathrm{L}= & \text { Peak, } 186 \mathrm{keV} \text {, detector } B \text {, left } \\ & \text { channel. }\end{aligned}$

P86' $R$ = Peak, $186 \mathrm{keV}$, detector $B$, right channel.

R86' $\mathrm{L}=$ High background, $186 \mathrm{keV}$, detector $B$, left channel. 
USE OF THE MENU

CHANGE ROIS?

(cont)
R86' $\mathrm{R}=$ High background, $186 \mathrm{keV}$, detector $\mathrm{B}$, right channel.

ONE BK REGION?

This question chooses between using one or two background regions to determine the Compton continuum under the 186-keV peak. Better results are obtained with one region on the high-energy side of the peak for low-resolution detectors. Answer YES.

ON HIGH SIDE?

Answer YES.

To change the ROI channel, enter its new value on the keypad and push ENT. To retain the present value, push ENT. After all ROI channels have been displayed, they are printed out (Fig. 8) and then written to EAROM.

\section{USE OF THE CRT}

CRT Features
The CRT displays the counts in each channel of the gamma-ray spectrum (Fig. 9). Channel numbers range from 0 to 1023. Channel zero is an exception and contains the elapsed time of the data acquisition. Along the baseline a dot indicates every tenth channel, and a bright dot indicates every hundredth channel. The spectrum from detector $A$ appears in chanrels 0 to 511 and the spectrum from detector $B$ appears in channels 512 to 1023. 
USE OF THE CRT

CRT Features

(cont) $\emptyset$

32768

$$
\begin{aligned}
& 28 \varnothing: 3 \varnothing 12 \\
& 250-34 \varnothing: 25 \varnothing_{1}
\end{aligned}
$$

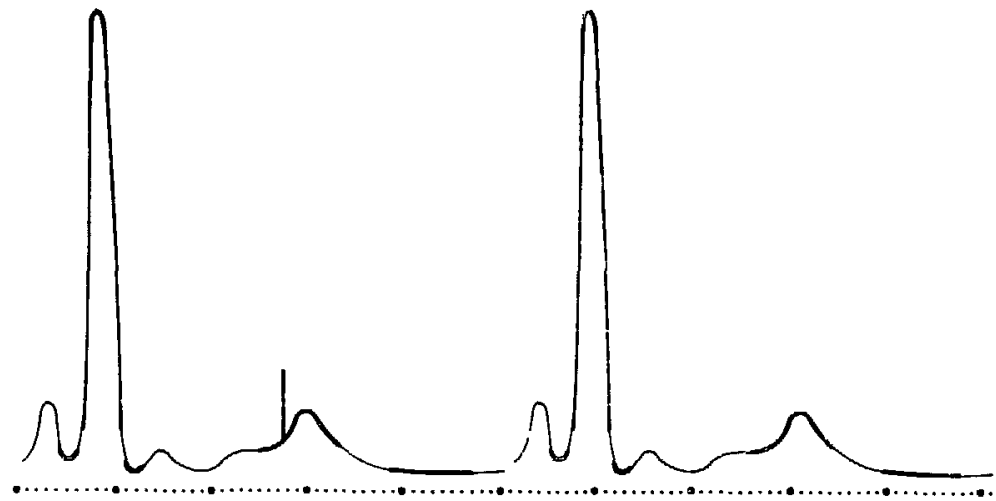

Fig. 9. Sample CRT display of the gamma-ray spectra. The 1024 channels are displayed with a vertical full scale of 32768 .

The numbers in the upper-left and -right corners indicate the left- and right-most channels that are displayed. An $x$-scale feature expands the $x$-axis by cycling in powers of 2, from 1024 to 64 channels. An offset feature allows the expansion to start at an arbitrary channel.

The number at the upper center, the maximum $y$-coordinate, indicates the vertical scale. (The maximum channel count is approximately threefourths of this value to avoid overlap with the alphanumerics.) In the default autoscale mode, the scale value is set at 8192 at the start of acquisition and increases automatically in powers of 2 as necessary. The $y$-axis can be expanded manually in powers of 2 by the $y$-scale feature. 


\section{USE OF THE CRT}

CRT Features

(cont)
ROIs used in the calculations of uranium concentration are intensified on the display. For example, in Fig. 9 the intensified ROIs are the ${ }^{241}$ Am photopeak ROI at $60 \mathrm{keV}$, its left and right background $\mathrm{ROIs}$, the ${ }^{235} \mathrm{U}$ photopeak $\mathrm{RO}$ : at 186-keV, and its right background ROI for each spectrum ( $A$ and $B$ ).

The cursor appears on the CRT display as a bright vertical bar. It can be moved left or right one channel at a time or to an arbitrary channel. At the upper center of the CRT display, the cursor channel is shown with the counts in that channel. In Fig. 9 the cursor is at channel 280, which contains the value 3012. If the cursor falls within a ROI, the boundary channels of that ROI are shown, along with the integral councs within the entire ROI. In Fig. 9 the ROI containing channel 280 covers channels 250 through 340 , and the integral within the ROI is 25001 .

During acquisition, the CRT disp? ay is refreshed approximately every $10 \mathrm{~s}$, or whenever a keypad entry changes the display.

CRT Operations from the Keypad
The following keys (Fig. 6) control the CRT display and are operational during data acquisition, except for the last $15 \mathrm{~s}$ of a cycle.

Moves the cursor one channel to the right. 


\section{USE OF THE CRT}

CRT Operations

from the Keypad

(cont)
Moves the cursor one channel to the left.

XSCL

Expands the $x$-scale and displays the left half of the current channels on the full horizontal scale. The $x$-range cycles in powers of 2 from 1024 down to 6.4 channeis, then back to 1024 .

YSCLL

Expands the $y$-scale by dividing the maximum channel count by 2 . If the YSCL key is pushed repeatedly, the maximum channel count cycles in powers of 2 down to 256 and then back to a maximum value of $2^{20}$.

AUTO

Resumes alitoscaling after the YSCL key has been used to change the $y$-scale.

CSR

Allows moving the cursor to an arbitrary channel. Enter the channel number when the LED display prompts CURSOR CHAN?.

OFFSET

Allows input of the left-most channel of the displayed expanded region. Enter the channel number when the LED display prompts OFFSET CHAN?. 


\section{ERROR MESSAGES}

Error Messages on the PDAC LED Display

The asterisk next to the uranium concentration indicates that a measurement control error occurred in the analysis of the most recent cycle. See the printout for details.

CYCLE TOO SHORT

This message is displayed at the completion of data acquisition if the analysis and/or output of results from the previous assay cycle are still in progress.

NO RUN COMPLETED

This message is displayed when the Look key is pressed if the first assay cycle has not yet been completed. Cycle counting begins at restart or if a YES answer is given to START ASSAY?. After cycle 1, this message indicates that mathematical errors prevented the completion of the assay cycle.

(No concentration display)

Normally, concentration is not displayed during cycle 1. If the conct.icration is not displayed after the completion of one or more cycles, mathematical errors have occurred. See the printout for details. 


\section{ERROR MESSAGES}

(cont)

Error Messages

on the Printout

\section{EAROM DESTROYED}

Some or all of the EAROM constants have been destroyed. Enter the devices, parameters, and ROIs as prompted before proceeding.

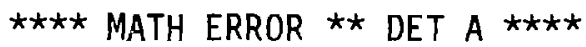

Some mathematical error has occurred in the computation of the detector A concentration result: division by zero, square root of a negative value, or logarithm of a nonpositive value. Any results not computed because of the problem appear as -8.888 on the printout.

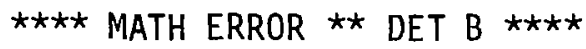

The meaning is the same as the previous description for detector $A$.

$\star \star \star \star$ PEAK POSITION IS BAD $\star \star \star \star ~$

The mean channel for the $60-\mathrm{keV}$ photopeak of the $A$ and/or $B$ spectrum is not within the window MEAN \pm DLTA.

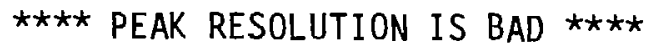
The resolution (100 - SIGMA/MEAN) of the 60-keV photopeak of the $A$ or $B$ spectrum is greater than RESL.

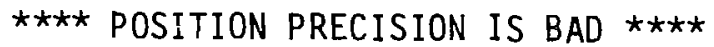
SIGMA of the mean channel for the $60-\mathrm{keV}$ photopeak of the $A$ and/or $B$ spectrum is greater than DLTA/3. 
THE CALIBRATION CURVE
The monitor determines the concentration of uranium by using the net area of the 186-keV photopeak (nomalized by the net area of the 60-keV photopeak). The shape of the calibration curve (uranium concentration vs count rate) is represented by a polynomial of the ninth order, determined by fitting calculated results obtained by numerical integration. The shape of the calibration curve depends on the location of the detector because effects of solution self-attenuation vary significantly in the wide range of solvent densities in the solyent extraction system, and because column diameters differ for glass and stainless steel sections. The monitor code stores up to 10 polynomial coefficients for each detector in the parameter file. Relocation of a detector almost always requires changing these parameters.

NORMAL IZING THE CALIBRATION
The normalization factor, NORM, is applied to the measured (normalized) 186-keV net photopeak area. The division by the 60-kev net area compensates for count-rate losses only. Determining normalization are the intrinsic NaI(TI) efficiency, the strength of the ${ }^{241}$ Am source, the source geometry, the $\mathrm{NaI}(\mathrm{TI})$ geometry within the collimator, composition and thickness of the solvent extraction column wall, and widths and positions of ihe ROIs used to define the photopeaks and backgrounds in the gamma-ray spectra.

To first order, all the factors comprising NORM are constant. However, in practice, a gradua? 
MORMAL IZING THE

CALIBRATION

(cont) upward drift in the normalization (of the order 0.5 to $1 \%$ per year) is observed. The drift is probably electronic in origin. Because the calibration curve is nonlinear, a $1 \%$ change in count rate corresponds to a 2 to $3 \%$ change in concentration in a concentration range of 100 to $200 \mathrm{~g}$ uranium per liter. Therefore, the normalization must be monitored regularly (monthiy) and adjusted when necessary.

The normalization is monitored by one of two methods. One method requires assay of a wellcharacterized uranium reference solution in the standard cell. The standard solution cell reproduces the geonetry of the solvent extraction coiumn. The other method uses a working standard uranium foil.

Normalization measurements for a new detector or for a detector that has been disassembled from the collimator shield and remounted in the same shield or in a new sinield are made using a reference solution in the standard cell. Thereafter, the uranium foil is used routinely to correct the normalization. However, reference solution measurements are recommended on a yearly basis to verify that the foil corrections are valid. The procedures for both the reference solution and the foil nomalizations are described separately below. 
DIRECT NORMALIZATION

MEASUREMENT WITH

REFEREACE SOLUTION IN

STANDARD CELL
The following steps are performed for one detector at a time:

(1) Rinse the standard cell once with the aque ous reference solution. (Typically, the uranium concentration is $100 \pm 50 \mathrm{~g} / \mathrm{l}$, and the acidity is very low. The 1300 or 2300 product solution is ideal.)

(2) Fill the standard cell with the aqueous reference solution. The detector must be mounted above the plates and rods that occupy the lower part of the standard cell. The solution height must be above the detector center by at least 4 in.

(3) Move the standard cell to the detector location at the solvent extraction column.

(4) Mount the detector onto the standard cell.

(5) Power up the printer and connect the RS-232 printer cable.

(6) Insert the tungsten shield into the detector collimator slot and run at least five background assay cycles.

(7) Verify that the average background ?2' (that is, $\overline{\mathrm{P} 2 \mathrm{~B}}$ ) is $0.0000 \pm 0.0002$. If necessary, adjust $D P-2(O L D)$, the background stored in 
DIRECT NORMALIZATION

MEASUREMENT WITH

REFERENCE SOLUTION IN

STANDARD CELL

(cont) the parameter file, as instructed in this Section under BACKGROUND MEASUREMENT AND ADJUSTMENT .

(8) Log all background results and the average, $\overline{\mathrm{P} 2 \mathrm{~B}}$, on the detector measurement control log sheet. Refer to the sample form, Fig. 10.

(9) Remove the shield and run 10 or more solution ssay cycles. Run at least 20 cycles for a new detector.

(10) At the end of the solution assay, withdraw three solution samples from the top of the cell for external assay, and obtain the average reference concentration, $\overline{\rho_{u}}$, the standard deviation, $\sigma\left(\rho_{u}\right)$, in these three restilts (in grams of elemental uranium per milliliter) and the \%RSD:

$$
\operatorname{rRSD}\left(\overline{\rho_{u}}\right)=100 \cdot \frac{\sigma\left(\rho_{u}\right)}{\overline{\rho_{u}}} \div \sqrt{3} .
$$

The \%RSD should be $<0.5 \%$. 


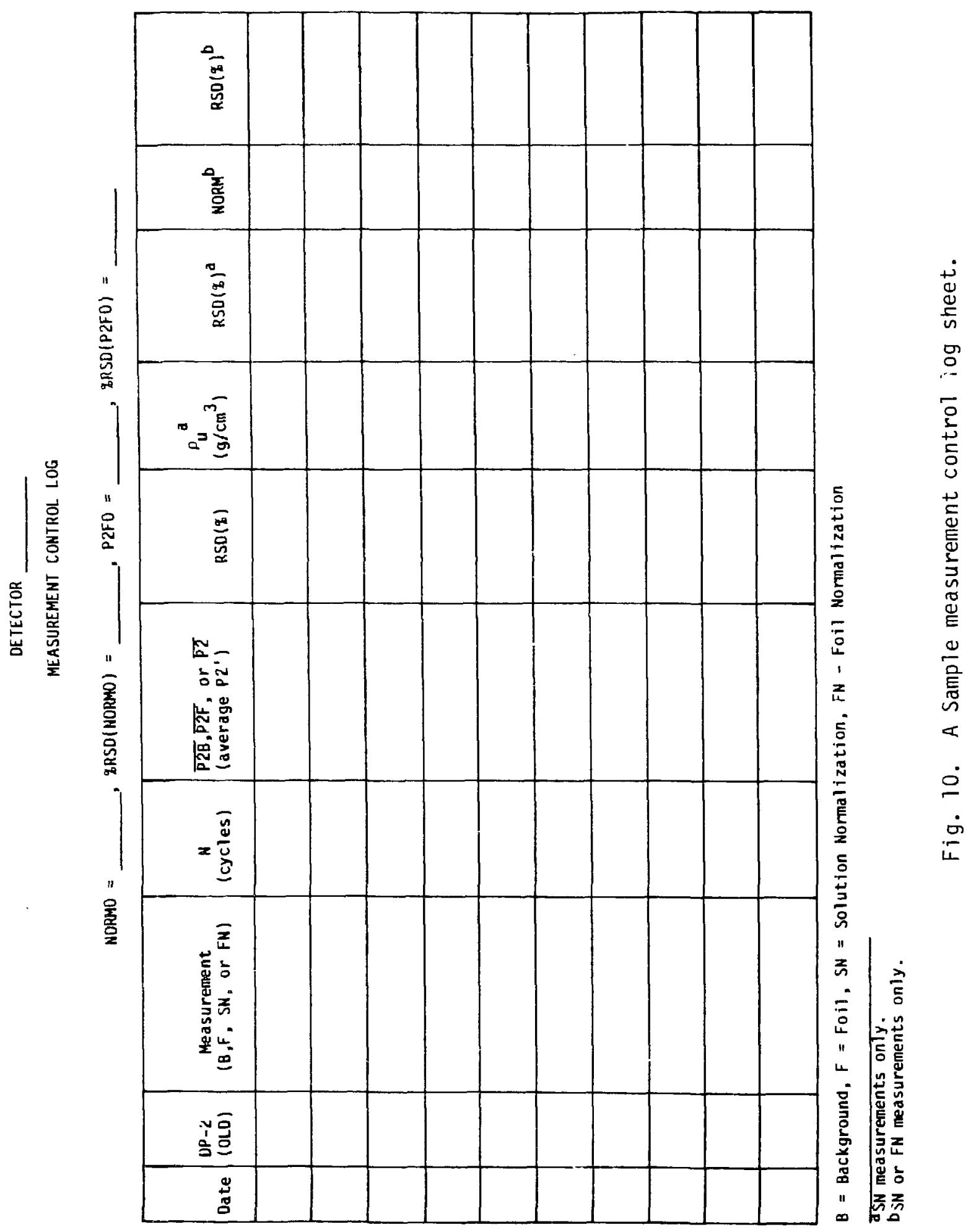


DIRECT NORMALIZATION

MEASUREMENT HITH

REFERENCE SOLUTION IN

\section{STANDARD CELL}

(cont)
(11) At the end of the solution assay, compute the average $P 2{ }^{\prime}$ from the $N(10$ or more) assay cycles (P2) and the \%RSD:

$$
\operatorname{gRSD}(\overline{\mathrm{P} 2})=100 \cdot \frac{\sigma(\mathrm{P} 2)}{\overline{\mathrm{P2}}} \div \sqrt{\mathrm{N}} .
$$

The \%RSD should be $<0.5 \%$.

(12) Using an interpolation of the appropriate table from Appendix $A$ (choose $M=0.2$ if the 1300 or 2300 product solution was used as the reference solution), obtain the expected count rate $C\left(\mathrm{~s}^{-1}\right)$ for the reference solution concentration, $\overline{P_{u}}$ (that is, RHOU, $\left.\mathrm{g} / \mathrm{cm}^{3}\right)$.

(13) Compute the new normalization value, NORM*: (a) Detector A (Organic, Extraction Column):

$$
\text { NORM }=\frac{\mathrm{C}}{\overline{\mathrm{P} 2}} \cdot 0.008573 .
$$

The constant, 0.008573, incorporates the attenuation effects of the glass column as well as the relative geometries for counting the 186- and 60-keV gamma rays.

*If normalization is for a new or remounted detector, tinen NORM $\equiv$ NORMG. 
DIRECT NORMALIZATION

MEASUREMENT WITH

REFERENCE SOLUTION IN

STANDARD CELL

(cont) (b) Detector B (Aqueous Product, Backwash Column ) :

$$
\text { NORM }=\frac{C}{\overline{P 2}} \cdot 0.009920
$$

The constant, 0.009920, incorporates the attenuation effects of the stainless steel column as well as the relative geometries for courting the 186and 60-keV gamma rays.

(14) Compute the \%RSD in NORM:

$$
\% \operatorname{RSD}(\operatorname{NORM})=\left[\% \operatorname{RSD}\left(\overline{\rho_{u}}\right)^{2}+\% \operatorname{RSD}(\overline{P 2})^{2}\right]^{1 / 2} .
$$

The \%RSD should be $<1 \%$.

(15) The new value of NORM should be within 1\% of the previously stored value (unless the detector is new or remounted).

(16) Log the values for:

- DP2(OLD), N, $\overline{\mathrm{P} 2}$, 㗁D $(\overline{\mathrm{P} 2})$,

- $\overline{\rho_{u}}, \operatorname{coRSD}\left(\overline{\rho_{u}}\right)$, and

- NORM, \%RSD (NORM)

on the detector $\log$ sheet. Refer to the sample form, Fig. 10. 
DIRECT MORMALIZATION

MEASUREMENT WITH

REFERENCE SOLUTION IN

STANDARD CELL

(cont)
(17) Enter the new value of NORM in the PDAC parameter file.

(18) Mount the detector in its normal position on solvent extraction column.

(19) Carry out steps 2-7 under NORMAL IZATION MEASUREMENT BY URANIUM FOIL ASSAY in this Section.

(20) Remove the foil from the detector slot.

(21) Power down the printer.

NORMAL IZATION MEASUREMENT BY URANIUM FOIL ASSAY
With the detector or detectors (both can be measured simultaneously) mounted in the normal position(s) on the solvent extraction $\operatorname{column}(s)$ :

(1) Power up the printer.

(2) Insert the tungsten shield(s) into the detector collimator(s) and run at least five background assay cycles.

(3) Verify the average background(s) $P 2^{\prime}$ (that is, $\overline{P 2 B})$ to be $0.0000 \pm 0.0002$. If neces sary, adjust the background( $s$ ) as instructed. (Refer to BACKGROUND MEASUREMENT AND ADJUSTMENT in this Section).

(4) Log the background results on the detector(s) measurement control $\log$ sheet(s). Refer to the sample form, Fig. 10. 
NORMAL IZATION MEASUREMENT

BY URANIUM FOIL ASSAY

(cont)
(5) Insert the uranium foil(s) into the detector collimator(s) and run at least $10^{*}$ foil assay cycles.

(6) Average the foil $\mathrm{P} 2$ ' results to get $\overline{\mathrm{P} 2 \mathrm{~F}}$. Obtain the \%RSD(s)** for the $\mathrm{N}$-cycle average ( $s$ ):

$$
\% R S D(\overline{P 2 F})=100 \cdot \frac{\sigma(P 2 F)}{\overline{P 2 F}} \div \sqrt{N} .
$$

The \%RSD should be $<0.5 \%$.

(7) $\log$ the foil results $\left[D P-2(O L D), N, \overline{r_{2} F}\right.$, $\% \operatorname{RSD}(\overline{\mathrm{P} 2 \mathrm{~F}})]$ on the measurement control $10 \mathrm{~g}$ sheet(s)of the detector(s). Refer to the sample form, Fig. 10. Plot the foil results. Refer to the sample form, Fig. 11.

(8) Compute the new normalization value(s), NORM:

$$
\text { NORM }=\frac{P 2 F 0}{P 2 F} \cdot \text { NORMO }
$$

*If the detector is new (or newly mounted), at least two sets (5 cycles per set) of foil measurements, with foil repositioning between sets, are recommended.

$\star \star$ If the detector is new (or newly mounted), then $\overline{\mathrm{P} 2 \mathrm{~F}} \equiv \mathrm{P} 2 \mathrm{~F} 0$, and $\% R S D(\overline{P 2 F}) \equiv \% R S D(P 2 F 0)$ for the foil $(s)$. 


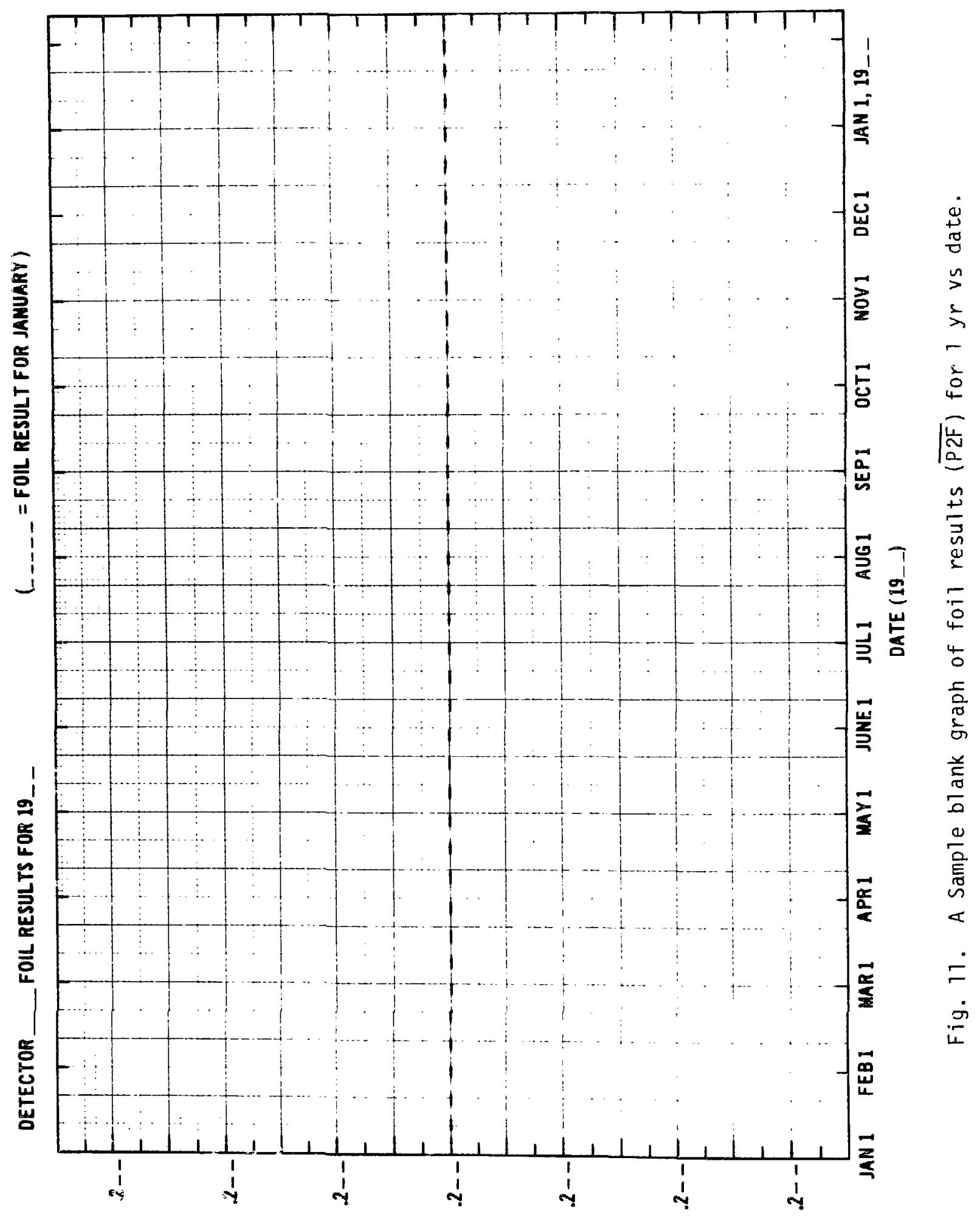


NORMAL IZATION MEASUREMENT

BY URANIUM FOIL ASSAY

(cont)

During the October 1983 calibration and installation, the following values were established:

Detector A NORMO $=15.28 \quad$ P2FO $=0.2388$

Letector $B \quad$ NORMO $=17.66 \quad$ P2F0 $=0.2499$

(9) Compute the \%RSD(s) in NORM:

$$
\begin{aligned}
\% \operatorname{RSD}(\mathrm{NORM})= & {\left[\% \operatorname{RSD}(\overline{\mathrm{P} 2 F})^{2}+\% \operatorname{RSD}(\mathrm{P} 2 \mathrm{FO})^{2}\right.} \\
& \left.+{ }_{\circ}^{\circ} \mathrm{RSD}(\text { NORMO })^{2}\right]^{1 / 2}
\end{aligned}
$$

The \%RSD should be $<1 \%$.

(10) The new value(s) of NORM should be within $1 \%$ of the previously stored value $(\mathrm{s})$.

(11) Install the new value(s) of NORM in the parameter file.

(12) Remove the foil(s) from the detector slot(s).

(13) Power down the printer.

BACKGROUND MEASUREMENT AMD ADJUSTMENT
Thie detector parameter file stores the background subtracted from al 1 measured values of the 186- to 60-keV net photopeak area ratio (P2) to give the background-subtracted ratio ( $\left.P 2^{\prime}\right)$. This ratio, $\mathrm{F} 2{ }^{\prime}$, has al so been corrected for ${ }^{241} \mathrm{Am}$ 
BACKGKOUND MEASUREMENT

AND ADJUSTMENT

(cont) decay. The background value is labeled DP-2 in the parameter file listing. The yalue of DP-2 must be evaluated and stored for a new (or remounted) detector. Each time measurement control is performed, DP-í is verified (and updated, if necessary). Under no circumstances should DP-2 exceed 0.002 , and in most cases it will be less than 0.001 .

If the measured DP-2 ever exceeds 0.001 , suspect that there is contamination on the collimator or the tungsten shield. Wipe these surfaces clean with alcohol, and repeat the measurements. In this discussion, DP-2(OLD) refers to the value of DP-2 stored in the parameter file, and DP-2(NEW) refers to the most recently measured value of DF -2 .

(1) Measure the background with the tungsten shield inserted in the detector collimator slot in five compiete background assay cycles. The average value of $P 2^{\prime}$ for the five cycles (that is, $\overline{\mathrm{P} 2 \mathrm{~B}}$ ) is typically $0.0000 \pm 0.0002$ if DP-2(OLD) is still valid.

(2) If

$$
|\overline{\mathrm{P} 2 \mathrm{~B}}|>0.0002
$$

compute the new value of DP-2:

$$
D P-2(N E W)=D P-2(O L D)+\overline{P 2 B} .
$$


BACKGROUND MEASUREMENT

AND ADJUSTHENT

(cont)
Store DP-2 (NEW) as DP-2, henceforth considered DP-2(OLD), in the detector parameter file.

(3) Record the new result for DP-2, DP-2(OLD) that is, the value stored in the parameter file at the time of the measurement), $N$, and $\overline{\mathrm{P} 2 \mathrm{~B}}$, in the detector measurement control $10 \mathrm{~g}$.

Once a week:

(1) Verify that no red i ights are i it (solid or flashing) on the NA-? 3 amplifiers. If red lights are lit, follcy the procedures described under ADJUSTMENT OF ANALOG ELECTRONICS in the EQUIPMENT SUMPIARY Section.

(2) Check the AGD and LED displays to verify proper operation of the display hardware.

(3) Check the results on the operator's LED displays during pulsed operation. Detector 1 (extraction column organic) assay should read $120 \pm 20 \mathrm{~g} / \mathrm{l}$. Detector 2 (backwash column aqueous product) assay should read $105 \pm 20$ $g / e$.

(4) Ensure that plastic tents are in place on detectors and that detectors are free of visible HEU contamination. 


\section{RECOMUENDED MEASUREMENT}

CONTROL PROCEDURES

(cont)

Monthly Procedure
Once a month or at least once every 4 months:

(1) Remove HEU contamination by wiping the column surfaces in front of the detectors, the Teflon end caps on the detectors, and the tungsten foil holders/shields.

(2) Verify (update, if necessary) the normalization factor for the calibration by uranium foil assay, described in NORMALIZATION MEASUREMENT BY URANIUM FOIL ASSAY in this Section.

(3) For all printed results, check that the 60-keV photopeak centroid (MEAN) and resolution (RESL) are within the acceptable limits, verified by the absence of an error message in the printout of results for each cycle. If MEAN is unacceptable, follow procedures described under ADJUSTMENT OF ANALOG ELECTRONICS in EQUIPMENT SUMMARY SEction to correct the problem. If RESL is unacceptable, replacement of the detector (described under ADJUSTMENT OF ANALOG ELECTRONICS in EQUIPMENT SUMMARY Section) might be nec sssary.

Yearly Procedure

Verify (update, if necessary) the normalization factor for the calibration by direct measurement with the reference solution in the standard cell, as described under NORMALIZATION MEASUREMENT WITH REFERENCE SOLUTION IN STANDARD CELL in this Section. 


\section{RECOMENDED MEASUREMENT \\ CONTROL PROCEDURES}

(cont)

Logging Measurement

Control Data
(1) Log all measurement control results on the measurement control $\log$ sheets. Figure 10 shows a sample log sheet.

(2) Plot all foil $\overline{\mathrm{P} 2 \mathrm{~F}}$ results vs date on a separate plot for each detector. Investigate any departures from a smooth monotonic dependence on time. Figure 11 is a sample blank graph.

(3) Log all procedures, including measurement control operations, maintenance, repairs, equipment replacements or adjustments of electronics settings, in chronological order in a lugbook dedicated to this instrument. The logbook will contain the original measurement contro? $\log$ sheets. 
OVERVIEW
The software automating the 2300 monitors consists of 53 FORTRAN and 33 assembly language subroutines. Table III lists the FORTRAN subroutines, organized by file, and briefly describes the function of each. Table IV is a similar list of the assembly language subroutines.

Figure 12 shows a simplified layout of structure levels within the code. The ASSAY subroutine executes data analysis and output of results. The FORTRAN subroutines most relevant to the data analysis and output functions are included in the ANALYZ and ASSAY files. Source listings of these eight subroutines are given in Appendix $B$. 
TABLE II I

FORTRAN SUBROUTINES

Filename: Subroutine: Function

ACQUIR: $\quad$ ACQUIR: Start data acquisition

ACWAIT: Wait for acquisition to finish, monitor keypad, ADC, CRT

SPDATA: Insert elapsed time (ET) into channel 0

SHOW : Show assay results and ET on LEDs

SHOWIT: Display line for $3 \mathrm{~s}$

SHOWRE: Show results on remote LEDS

ALPHA : $\quad$ ALPHAX: Convert a real number to ASCII

ALPHAI: Corvert an integer number to ASCII

NUMBER: Convert ASCII number to real

ANALYZ: ANALYZ: Uranium concentration analysis

POLYNM: Compute a polynomial

PEAKS : Compute peak areas, sigmas; initialize mean and resolution flags

TESTS : Measurement control (MC) tests

AREA : Determine net area from three ROIs

ASSAY : ASSAY : Do repeated assays

OIJTPT1: Print ROI sums, net areas

OUTPT2: Print corrected areas, MC results, uranium concentration, error messages

CHANGE: MODCON: Modify constants

CHGPLY: Change polynomial coefficients

MODROI: Modify ROI

CHANGX: Change a real number

CHANGI: Change an integer number

MCDDEV: Modify device status

PRTCON: Print constants 
TABLE III (cont)

Filename: Subroutine: Function

\begin{tabular}{|c|c|c|c|}
\hline & & PRTPLY: & Print polynomial coefficient \\
\hline & & PRTBK : & Print number background ROIs for each peak \\
\hline & & ENTRSG: & Enter specific gravity \\
\hline CRTSUB & & CRTINP: & Interpret keypad input for CRT \\
\hline & & KEFRSH: & Refresh CRT \\
\hline & & CURSOR: & Display cursor and cursor information \\
\hline & & INICRT: & Initialize parameters for CRT \\
\hline & & STRCRT: & Initialize CRT for a new run \\
\hline DATTIN & & DATTIM: & Retrieve or change date and time \\
\hline & & JULDAY: & Compute Julian day from $10 / 30 / 79$ \\
\hline MAIN & : & MAIN : & Execute menu and calls to high-level routines \\
\hline & & INITL : & Initialize constants on power up/restart \\
\hline READ & : & READX : & Prompt user and read a real number \\
\hline & & READI : & Prompt user and read an integer \\
\hline & & ACCEPT: & Accept numeric input from keypad \\
\hline & & QUESTN : & Ask $Y / N$ question and wait for response \\
\hline & & FLASH : & Flash a message on LED panel and wait for input \\
\hline ROI & : & GETROI: & Get ROI sum \\
\hline & & INTROI: & Intensify ROIs \\
\hline UTIL & : & ROUND : & Round real number to fit a format \\
\hline & & DUMPSP: & Dump spectrum to printer \\
\hline & & OPENLP: & Open LP RS232 \\
\hline & & CLOSLP: & Close LP RS232 \\
\hline & & REPROM: & Read EEProM \\
\hline & & WEPROM: & Write EEPROM \\
\hline & & OPN422: & Open LP RS422 \\
\hline & & CLS422: & Close LP RS422 \\
\hline & & PALARM: & Turn on precipitation alarm light \\
\hline & & USTRIP: & Put out uranium grams/1iter to strip chart \\
\hline
\end{tabular}




\section{TABLE IV}

ASSEMBLY LANGUAGE SUBROUTINES

Filename: Subroutine: Function

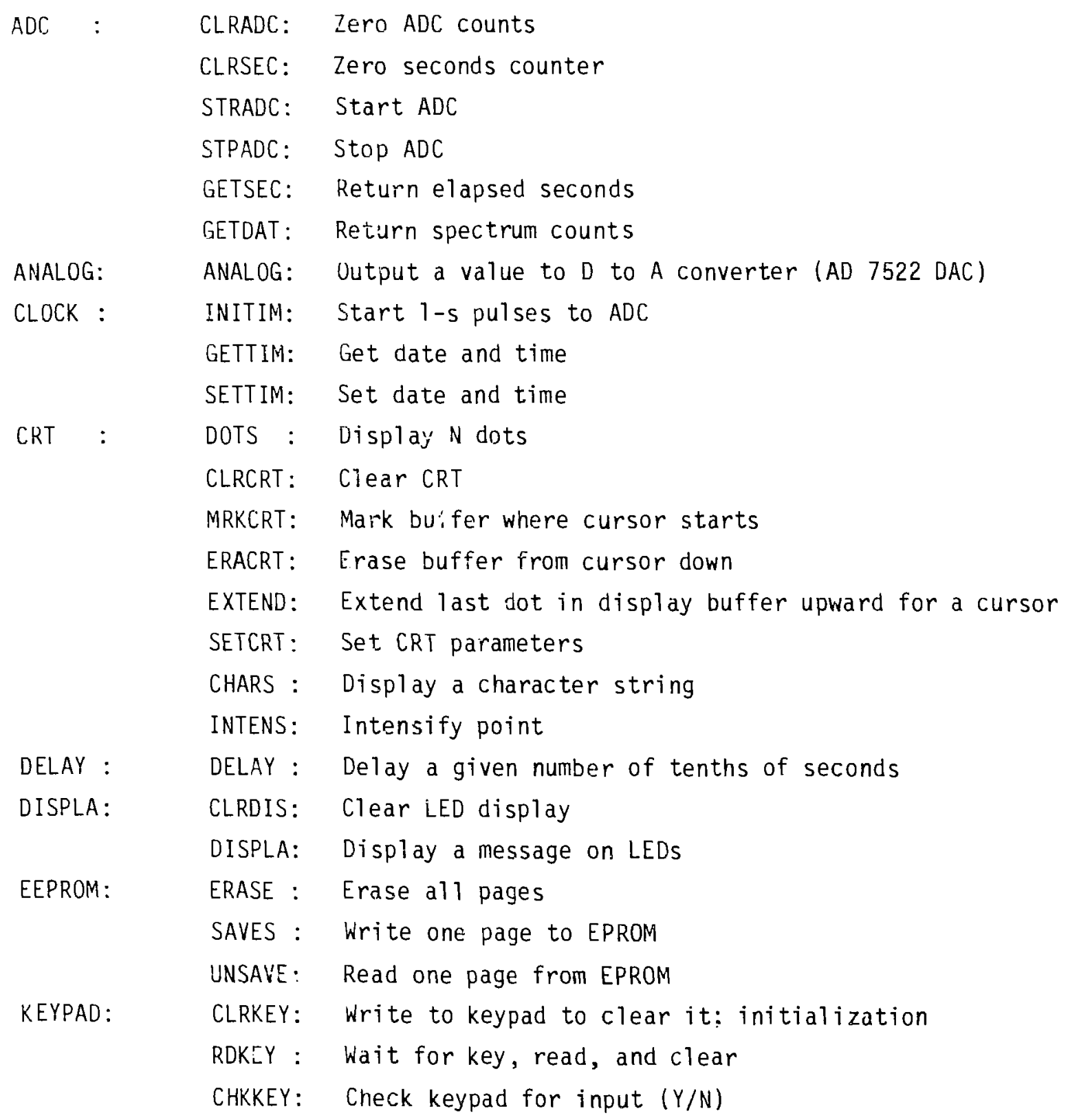


TABLE IV (cont)

Filename: Subroutine: Function

LIGHTS: $\quad$ LGTOFF: $\quad$ Driver precipitation alarm lights

LIGHT : Driver precipitation alarm lights

BAROFF: Driver concentration bar chart lights

BAR : Driver concentration bar chart lights

PRINTR: $\quad$ PRINTR: FORTRAN-callable device driver for serial APP-48 printer

REMDIS: REMDIS: Driver for remole displays

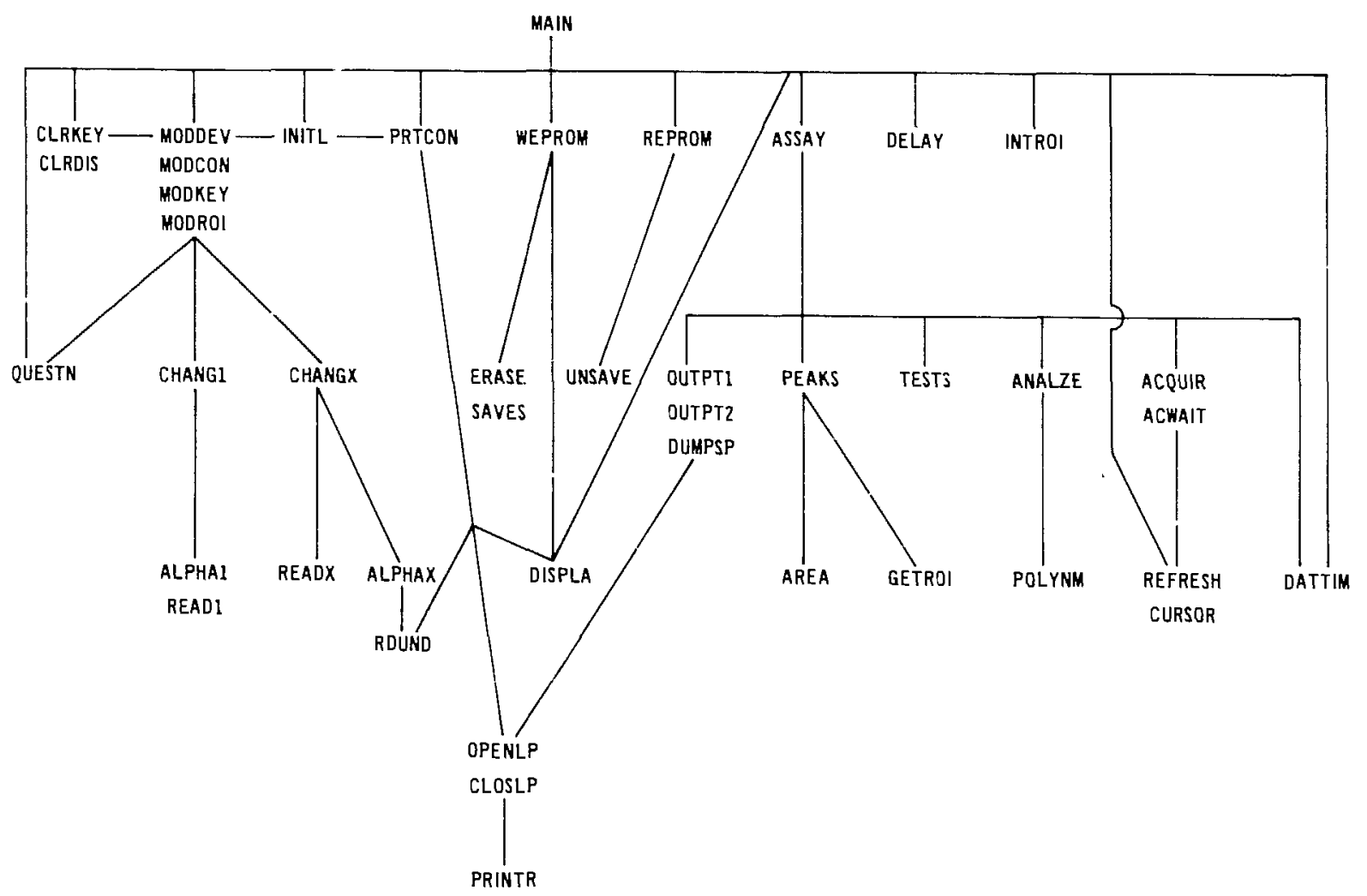

Fig. 12. The layout and calling sequences in the program for the 2300 monitors. 
The concentration monitor for the aqueous product of the backwash coilumn was evaluated over a 2month period. The monitor results were compared with the assays of samples removed from the product stream and analyzed using a high-resolution gamma-ray transmission method. ${ }^{7,8}$

Figure 13 shows the evaluation results for the monitor and external sample assays. The 10 error bars $( \pm 1.3 \%)$ on the monitor results give the predicted uncertainty of the 100-s monitor assay based on counting statistics. The 10 error bars $( \pm 0.7 \%)$ on the external assay results give the estimated net random uncertainty in the results of the external assay. The monitor results agree with the external assays within the measurement uncertainties.

Similar evaluation data for the concentration monitor of the organic product of the extraction column hav: not been obtained yet. The monitor was initially installed above but near the phase interface to include a significant (visible) fraction of aqueous phase. Therefore, it is not possible to withdraw a representative sample for comparison. Although this detector location is satisfactory for the needs of process monitoring (the monitor assays the average uranium concentration in the two-phase mixture), evaluation of this monitor must be performed on static, singlephase solutions to circumvent the sampling difficulties. 


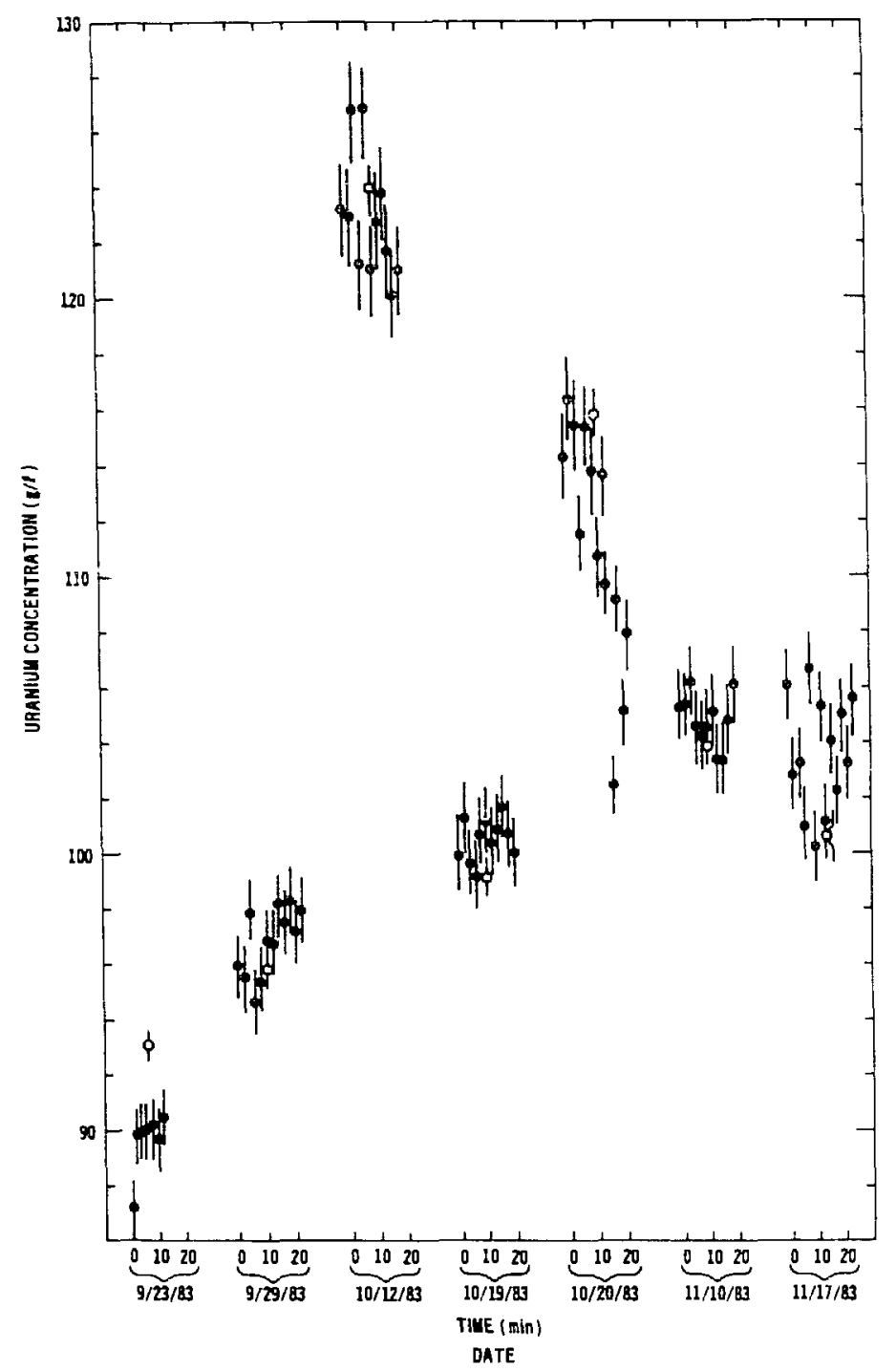

Fig. 13. Uranium concentration is plotted vs time on specific dates during the evaluation of the backwash column aqueous product monitor. The solid points are the monitor assay results for successive 100-s assay cycles. The open circles are the results of external assay of samples withdrawn from the aqueous product stream. The open circles are plotted it the time of sample withdrawal. Error bars on the open circles correspond to the estimated uncertainty $(1 \sigma)$ in the external assay. Error bars on the solid points are the 10 values computed from the counting statistics of the 100-s monitor assay. 
SUMMARY

(cont)
Shutdown of the 2300 solvent extraction system to install automatic control capabilities in 1984 has caused a postponement of the evaluation of the concentration monitor on the organic product of the extraction column until the system is again operational. 


\section{REFERENCES}

1. P. A. Russo and R. B. Strittmatter, "On-Line Prototype NDA Instruments for Material Accounting and Monitoring of HEU Recovery Processes," Trans. Am. Nuc1. Soc. 43,274 (1982).

2. P. A. Russo, R. B. Strittmatter, E. L. Sandford, I. W. Jeter, E. McCullough, G. L. Bowers, "Operation of Automated NDA Instruments for In-Line HEU ACcounting at $Y-12$," in "Proceedings of the ANS/INMM Conference on Safeguards Technology: The Process-Safeguards Interface," Hilton Head Island, South Carolina, Ncvember 28-December 2, 1983, USDOE New Brunswick Laboratory, Conf. No. 831106 (August 1984), pp. 112-134.

3. T. L. Brumfield, S. E. Smith, E. E. McCullough, and P. Russo, "On Line Operation of NDA Instrumentation on a Solvent Extraction System," Martin Marietta Energy Systems, Inc., Oak Ridge Y-12 Plant report Y/DG-16602, 1984.

4. P. A. Russo, R. B. Strittmatter, E. L. Sandford, M. M. Stephens, T. L. Brumfield, S. E. Smith, E. E. McCullough, I. W. Jeter, and G. L. Bowers, "Al:tomated Instruments for In-Line Accounting of Highly Enriched Uranium at the Oak Ridge Y-12 Plant," Los Alamos National Laboratory report LA-10243-MS (to be pub1 ished).

5. EUREKA Operation Manual, Los Alamss National Laboratory document (Q-1-84-724).

6. R. S1 ice, "PDAC-100 Operations Manual," Los Alamos National Laboratory report LA-10383-M (to be published).

7. I. W. Jeter, S. E. Smith, H. H. Hogue, G. L. Bowers, P. A. Russo, and R. B. Strittmatter, "Applications of NDÁ Instrumentation in the Y-12 Highly Enriched Uranium Recovery Facility," in "Proceedings of 0-lando International AICHE Meeting, Orlando, Florida (1982)," available in microfiche from AICHE.

8. H. H. Hogue and S. E. Smitin, "Off-Line Nondestructive Assay at a Uranium Recovery Facility," in "Proceedings of ANS/INMM Conference on Safeguard: Technology: The Process-Safeguards Interface," Hilton Head Is Iand, South Carolina, November 28-December 2, 1983, USDOE New Brunswick Laboratory, Conf. No. 831106 (August 1984), pp. 265-274. 


\section{CALCULATED 186-keV COUNT RATES* VS ELEMENTAL URANIUM CONCENTRATION}

AQLIEDUL:

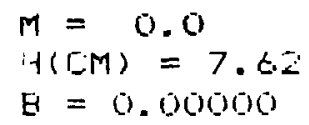

FiHoil_I

( $G / E M B)$

.02100

.02200

.02300

.02400

.02500

.02600

.02700

.02800

.02800

.03000

$.03100^{\circ}$

.03200

. 05000

.03400

.03500

.03600

.03700

.03800

.03500

.04000

.04100

.04200

.04300

.04400

.04500

.04600

.04700

.04500

.04900

.05000

$$
\begin{gathered}
{[(\text { RHLIUI, } 0.0)} \\
(S E D-1)
\end{gathered}
$$

$9.50 \% 2062 E+01$

$7.70 E 6026 \mathrm{E}+01$

1. $03035 \% 4 E+02$

1. $0641 E G E+02$

1. $10 E 0445 E+02$

1. $1462456 E+02$

1. $1840215 E+02$

1. $221375 \mathrm{BE}+02$

i. $2535275 E+02$

1. $2345645 E+02$

1. $3310011 E+02$

1. $: 0607363 \mathrm{E}+02$

1. $4020774 E+02$

1. $4370315 E+02$

1. $4715983 E+02$

1. $5057045 E+02$

1. $5.95 \% 6 \%+02$

1. $5730352 E+02$

1. $60<130 E E+02$

1. $636 \mathrm{~B} 219 \mathrm{E}+02$

$1.6711773 E+02$

1. $7031773 \mathrm{E}+02$

1. $7348143 E+02$

1. $760.121 \% E+02$

1. $7970 \% 00 E+02$

1. $3277005 E+02$

1. $5: 57 \% 8 \% 4 E+02$

1. $E E 7 \% 435 E+02$

1. $51756 x+7 E+02$

1.94@8727E+02

*Applies to $Y-12$ 3.75-in.-i.d. stainless steel cell with no plates. Column attenuation of gamma rays is not included in the calculation. Assumes uranium enrichrient of the 9212 facilities. 
FituOII

( $\mathrm{G} / \mathrm{CH}$ (3)

.05100

.05200

. 05300

.05400

- 05500

.05600

.05700

.05800

$.05 \% 00$

.06000

.06100

.00200

- 06300

.06400

.06500

.06 .600

.06700

.06500

.06500

.07000

.07100

.07200

.07300

.07400

.07500

.07600

.07700

.07800

.07900

. 08000

- OE100

. 05200

.08300

.03400

.08500

. 08600

. 08700

- OBs0

- 0E500

.09000

. 09100

.07200

- 0\%300

.09400

.08500

.09600

$.0 \% 700$

.09800

- 09900

.10000
C: (RHCIU, O.O)

(SE:-1)

1. $9755579 E+02$

$2.0045316 E+02$

2. $0323576 E+02$

2. $0609416 E+0^{-}$

2. $0696 \% 4.7 \mathrm{E}+\mathrm{C}$

2. $11612.7 \mathrm{E}+\mathrm{O}$

$\therefore 14327 \% 6 E+02$

$2.17013005+02$

2. $196.921 \mathrm{E}+02$

2. 22\%6\%E+02

2. 24586.17E+0

․ $274665 \mathrm{E}+02$

2. $300100 \mathrm{E}+02$

$2.32525=02$

$2.3501420 E+02$

$2.3747602 E+02$

$2.99 \%: 141 E+02$

$2.4232044 \mathrm{E}+02$

2. $447030 \bar{E}+02$

2. $4706121 E+02$

2. $4957397 E+02$

$2.5170147 E+02$

2. $53533 \% E+02$

2. $5624198 E+52$

$2=5347609 E+12$

2. $6065627 E+02$

2. $625727 ? \mathrm{E}+02$

$2.6503544 E+02$

$2.6717619 \mathrm{E}+2$

$2.69293 \mathrm{ES}+02$

$2.7130791 \mathrm{E}+02$

$2.7346095 \mathrm{E}+02$

$2.7551076 \mathrm{E}+02$

$2.7754001 \mathrm{E}+02$

$2.7954737 E+02$

2. $015330 \mathrm{O}+0 \%$

2. $8349510 E+02$

$2.854420 E+02$

$2.8736564 E+02$

2. $8 \% 26896 E+02$

2. $9115239 E+02$

$2.93015215+02$

2. $9455510 E+02$

$2.953330 E+02$

$2.9348550 \mathrm{E}+02$

$3.0027456 \mathrm{E}+02$

3. $0204185 E+02$

S. $0379131 E+02$

3. $05.52078 E+02$

$3.0723367 E+02$

R. 2 
FiHLIII
$($ G/ENI

.10100

.10200

.10300

.10400

.10500

.10600

.10700

.10300

.10900

.11000

.11100

.11200

.11300

.11400

.11500

.11600

.11700

$.11 \mathrm{G00}$

.11900

.12000

.12100

. 12200

.12300

.12400

.12500

- 12600

.12700

- 12800

. 12900

.13000

. 13100

. 13200

.13300

. 13400

.13500

.13600

.13700

. 13900

.13900

.14000

.14100

.14200

.14300

.14400

.14500

.14600

.14700

.14300

.14900

.15000
C: $($ RHLILI, 0.0) ( $E E E:-1$ )

3. $0852516 E+02$ ‥ $1060489 \mathrm{E}+02$ S. $1226446 E+02$ 3. $1390634 E+02$ $3.155004 \mathrm{E}+02$ $3.1713962 E+02$ 3. $18,3060 E+02$ 3. $2030527 E+02$ 3. $2186346 E+02$ $3.2340532 E+02$ $3.24 \% 3212 \mathrm{E}+02$ 3. $2644273 \mathrm{~B}+02$ $5.2793756 E+02$ 3. $2 \% 41674 \mathrm{E}+02$ I. $3059103 \mathrm{E}+02$ 3. $3233037 \mathrm{E}+02$ $3.3376 .450 E+0=$ 3. $3518370 \mathrm{E}+02$ 3. 365EE:5E+02 $3.37 \% 7 \% 25 E+02$ 3. $3935520 E+02$ $3.4071763 \mathrm{E}+02$ $3.4206596 E+02$ 3. $434002 \mathrm{EE}+02$ $3.4472077 \mathrm{E}+02$ 3. 46.02524E+02 3. $4732205 \mathrm{E}+02$ 3. $4960301 \mathrm{E}+02$ 3. $4587100 \mathrm{E}+02$ $3.5112625 E+02$ 3. $52367 \% 6+02$ 3. $5359780 E+02$ 5. $54814 \mathrm{EE}+0$ $3.5601 \%$ ECE +02 3. $572125 \mathrm{EE}+02$ 3. $5839346 E+02$ B. 5 .5口2 I EE +02 $3.607192 \mathrm{EE}+02$ $3.6186480 \mathrm{E}+02$ $3.6299080 E+02$ 3.6.412125E+02 3. $5523262 E+02$ $3.66332 \% 1 E+02$ 3. $6.742235 E+02$ $3.6550050 E+02$ $3.6756323 E+02$ 3.706.53E+02 3. $716719 \% \mathrm{E}+02$ $3.7270789 E+02$ $3.737331 \mathrm{E}+02$ 
FHCILI

( $\mathrm{G} / \mathrm{C} N \mathrm{~N} 3$ )

.15100

.15200

. 15300

.15400

. 15500

- 15600

.15700

.15800

.15900

.16000

.16100

.16200

. 16300

.16400

.16500

.16600

.16700

.16800

.16900

.17000

.17100

.17200

.17300

.17400

.17500

.17600

.17700

.17800

.17900

.18000

- 18100

. 18200

.18300

.13400

.18500

.18600

.18700

- 18800

.18900

.17000

.19100

- 19200

.19300

.19400

- 19500

.19600

.19700

.19300

.19700

.20000
C: (RHEIU,, 0.0$)$

( $S E E L-1$ )

$3.7474943 E+02$

$3.7575515 \mathrm{E}+02$

3. $767510 \mathrm{EE}+02$

$3.7773690 \mathrm{E}+02$

$3.7871301 \mathrm{E}+02$

$3.7967946 E+02$

$3.8063670 E+02$

$3.8158449 E+02$

3. $5252284 E+02$

3. $8345216 E+02$

$3.843720 \% E+02$

$3.3520300 \mathrm{O}+02$

3. 86185G1E+02

3. $5707917 E+02$

$3.8796409 E+02$

3. BES4011E+02

$3.8770792 E+02$

$3.9056703 E+02$

3. $\$ 141 \mathrm{B34E+02}$

$3.9226087 E+02$

3. $9309574 E+02$

3. $939224 E+02$

$3 . \$ 47411 \mathrm{UE}+02$

$3.9555193 \mathrm{E}+02$

$3.56 .35476 . E+02$

$3.9715012 \mathrm{E}+02$

$3.9795795 E+02$

$3.9571512 E+02$

3. 94\%114E+0Z

4. $0025029 E+02$

4. $0101446 E+0$

4. $0176537 E+02$

4. $0250 \% 22 E+02$

4. $0324595 E+02$

4. $0: 97577 E+02$

4. $046.9567 E+02$

4. $0541484 \mathrm{E}+02$

4. $0612420 E+02$

4. $0652675 E+02$

4. $075230 \% E+02$

4. $0821266 E+02$

4. $0839575 E+02$

4. $0 \% 57250 E+02$

4. 1024300E+0Z

4. $1030728 E+02$

4. $1156537 E+02$

4.1221717E+0Z

4. $1266295 E+02$

4. $13502 y=E+02$

4. $141370 E E+02$

A. 4 
FiHOII

( $6 / 2 . M B)$

.20100

.20200

.20300

.20400

.20500

20600

.20700

20300

20900

.21000

.21100

. 21200

.21300

.21400

.21500

.21600

.21700

.21500

.21900

. 22000

.22100

.22200

.22300

22400

. 22500

22600

.22700

- 2800

22900

.23000

.23100

- 23200

.23300

. 23400

- 23500

.23600

. 23700

. 23800

.23900

.24000

.24100

.24200

.24300

.24400

. 24500

.24600

.24700

. 24800

.24500

.25000
C: (RHCILI, 0.0)

( $S E E:-1$ )

$4.1476 .525 E+02$

4. $1535765 E+02$

4. $160042 E E+02$

4. $1601529 E+02$

4. $1722067 E+02$

4. $17620.54+02$

4. $1941499 E+0=$

4. $1 \% 00 \% \mathrm{E}+02$

4. $195757 \mathrm{E}+0 \mathrm{0}$

4. $2016577 E+02$

$4.2073594+02$

4. $2150675 E+02$

4. $2156956 . E+02$

4. $2242714 E+02$

4. $22979620+02$

4. $2352737 E+02$

4. $2407015 E+02$

4. $2460307 E+02$

4. $2514117 E+0$

4. 2SGSOSOE+O2

4. $26.19312 E+02$

4. $2071201 E+02$

4. $2722636+02$

4. $2773011 E+02$

4. $2324135 E+02$

4. $2374212 E+02$

4. $292355 E+02$

4. $27705 \mathrm{E}+02$

4. $3021 \mathrm{SOOE}+02$

4. $3070161 \mathrm{E}+02$

4. $3115072 E+02$

4. $316.556 E E+02$

4. $3212644 E+02$

4. $325 \% 3 E+02$

4. $33055 E 4 E+02$

4. 3351 455E+02

4. $3396926 E+02$

4. $3441 \% \%$

4. $34566 \% E+O 2$

4. $35094 \mathrm{E}+02$

4. $35748 \% E+02$

4. $3610447 E+02$

4. $36.6 .16 .23 E+02$

4. $3704431 E+U 2$

4. $3746.8: 71 E+02$

4. $3785 \% 4 \dot{C}+02$

4. $35: 0.55 E+02$

4. $3572025 E+02$

4. $3913034 E+02$

4. $3950716 \mathrm{E}+02$ 
RHOLLI

(LILMIS)

.25100

.25200

. 25300

.25400

. 25500

.25000

. 25700

.25800

.25900

. 26000

. 26100

.26200

.26300

. 26400

. 26500

26600

26700

. 26800

26900

.27000

. 27100

.27200

.27300

.27400

.27500

.27500

.27700

.27800

.27900

- 28000

. 28100

.28200

.28300

. 28400

.28500

.28600

.25700

.26300

28900

.27000

. 27100

.29200

.29300

.29400

.27500

. 29600

.28700

29800

.29900

.90000
C: (RHLU, 0.0) (SEL:-1)

4. $3994027 E+02$ 4. $403401 \mathrm{E}+02$ 4. $4073604 E+02$ $4.4112977 \mathrm{E}+02$ 4. $415176.6 E+02$ $4.4190635 E+02$ 4. $4225851 \mathrm{E}+02$ 4. $4260894 E+02$ 4. $4304707 E+02$ 4. $4342101 E+02$ 4. $437918 \mathrm{EE}+0 \mathrm{~S}$ 4. $4415064 E+02$ 4. $4452446 E+02$ 4. $4435620 E+02$ 4. $45.2506 \mathrm{O}+02$ 4. $456000 \mathrm{BE}+02$ 4. $45.53 \% 4 E+02$ 4. $4630401 E+02$ 4. $400.512 \% E+02$ 4. $469.9577 E+02$ 4. $4735740 E+02$ $4.476763 \mathrm{E}+02$ 4. $4501250 \mathrm{E}+02$ 4. $4334605 E+02$ 4. $486.7675 E+02$ 4. $4700472 \mathrm{E}+02$ 4. $4933052 E+02$ 4. $4965342 E+02$ 4. $4997590 \mathrm{O}+02$ 4. $502 \% 167 E+02$ 4. $50600.92 E+02$ 4. $50 \% 1979 E+02$ 4. $5125007 E+02$ 4. $515.3007 E+02$ 4. $5184357 E+02$ 4. $5214662 E+02$ 4. $5244736 E+0$ 4. $52745 \mathrm{EE}+02$ 4. $5304134 \mathrm{E}+02$ 4. $533566 E+02$ 4. $5362717 E+02$ 4. $5.31641 E+02$ 4. $5420342 E+02$ 4. $54433=2 E+02$ 4. $547709 E+02$ 4. $550.5141 \mathrm{E}+02$ 4. $5532975 E+02$ 4. $556059 \%+02$ 4. $5.585007 E+02$ 4. $5 E .15216 E+02$

A. 6 
AQLIEOISS

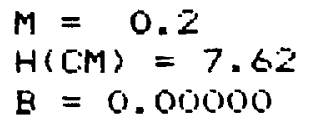

FiHCIII

( $0 / \mathrm{CM} 3$ )

.01100

.01200

.01300

.01400

.01500

.01600

.01700

. 01800

.01900

.02000

.02100

. 02200

.02300

.02400

.02500

.02600

.02700

.02800

.02900

.03000

.03100

.03200

.03300

.03400

.03500

.03600

.03700

- 05800

.03700

.04000

.04100

.04200

.04300

.04400

.04500

.04600

.04700

.04800

.04500

. 05000
C. (FIHEHI, 0.2)

(SEE:-1)

5. $2540794 \mathrm{E}+01$

5. $7003101 E+01$

$6.141546 t E+01$

6. 577 BEBSE+01

7. $00 \% 500 E+01$

$7.4300312 E+01$

$7.575 / 457 E+01$

B. 2751630E+01

E. 6577791E+01

7. $0.5755 C E+01$

\%. 4 S. 2E:7EE+01

7. $3 \% 14 E+01$

1. $02 \% 2871 E+02$

1. $0630104 E+02$

1. $106.592 \mathrm{E}+02$

1. $1450635 E+02$

1. $1829032 E+02$

1. $2201294 \mathrm{E}+02$

1. $2570357 E+02$

1. $2935415 \mathrm{E}+02$

1. $396442 E+02$

1. $36.5342 \angle E+02$

1. $4006572 E+02$

1. $4355767 E+02$

1. $4701119 E+02$

1. $5042696 E+02$

1. $5.304 \% 2 E+02$

1. $5714760 E+02$

1. $6045257 E+02$

1. $6371917 \mathrm{E}+02$

1. $6495206 E+02$

1. $7014363 \mathrm{E}+02$

1. $7331020 E+02$

1. $7643794 \mathrm{E}+02$

1. $795.317 \% \mathrm{E}+02$

1. $8259111 \mathrm{E}+02$

1. $55.61630 E+02$

1. $8 \mathrm{BE} 0994 \mathrm{E}+02$

1. $9157041 E+02$

1. $9447805 E+02$ 
RHCU

( $5 / \mathrm{CM} 3)$

.05100

.05200

. 05300

. 05400

. 05500

- 05600

.05700

.05800

.05900

. 06.000

- $0 \leqslant 100$

.06200

- 06500

. 06400

. 06500

. 06800

.06700

. 06800

.06900

.07000

.07100

.07200

.07300

.07400

.07500

.07600

.07700

.07800

.07500

.08000

.08100

.08200

.08300

- 08400

- 0E500

- 0E600

- 05700

.08500

- 05900

.09000

.09100

.09200

.08300

.09400

.09500

$.0 \% 600$

.09700

.08500

.09800

.10000
C(RHOL, 0.2$)$

(SEC-1)

1. $9735440 E+02$

2. $0025936 E+02$

$2.030 \% 300 E+02$

2. $058.569 E+02$

2. $0566.7 \% 6 E+02$

2. $1141097 E+02$

2. $1412287 E+02$

2. $1680612 \mathrm{E}+02$

$2.1946023 E+02$

2. $2208667 E+02$

2. $246 E 292 E+02$

2. $2725222 E+02$

2. $2579575 E+02$

$2.3230727 E+02$

2. $3479426 E+02$

2. 372546\%E+02

2. 3968787E+02

2. $420756.7 E+02$

2. $4447762 \mathrm{E}+02$

2. $4683292 E+02$

2. $4916400 E+02$

$2.5146 .970 E+02$

2. $5375110 \mathrm{E}+02$

2. $560070 \mathrm{BE}+02$

2. $5823884 \mathrm{E}+02$

2. $604487 E E+02$

$2.6263428 E+02$

$2.64795 \Leftrightarrow 7 E+02$

2. $669343+5 E+02$

$2.6905036 E+02$

2. $71143 \mathrm{EE}+02$

2. $732153 \mathrm{BE}+02$

2. $7526474 \mathrm{E}+02$

$2.7729227 \mathrm{E}+02$

$2.7929794 \mathrm{E}+02$

2. $512=30 \mathrm{EE}+02$

2. $8324754 \mathrm{E}+02$

2. E5ः $8937 E+02$

$2.8711246 E+02$

2. $3901407 E+02$

2. $9059705 E+02$

2. $7275744 E+02$

2. $9460211 E+02$

$2.7642552 E+02$

2. $9322969 \mathrm{E}+02$

3. $000147 \mathrm{SE}+02$

3.017E125E+02

$3.0352937 \mathrm{E}+02$

3. $0525911 E+02$

$3.0697101 E+02$

A. 8 
FHCIU

(G/CMB)

.10100

.10200

.10300

.10400

.10500

.10600

.10700

.10800

.10900

.11000

.11100

.11200

.11300

.11400

.11500

.11600

.11700

.11800

.11500

.12000

.12100

.12200

.12300

.12400

.12500

$-12600$

.12700

.12800

.12900

.13000

.13100

.13200

.13300

.13400

.13500

. 15000

.13700

.13800

.13900

.14000

.14100

.14200

.14300

.14400

.14500

.14600

.14700

.14500

.14900

.15000
C(FIHOL, 0.2$)$

(SEL-1)

3. $0566424 E+02$

3. $1034041 \mathrm{E}+02$

3.119991BE+OZ

3. $1304024 \mathrm{E}+02$

3. $1523432 E+02$

3. $163719 \mathrm{EE}+02$

3. $1546226 E+02$

$3.2003658 E+02$

3. $2159435 E+02$

ज. $2313621 E+02$

$3.2466192 E+02$

$3.201716 \% E+02$

$3.27665 \% 7 \mathrm{E}+02$

$3.291449 \mathrm{GE}+02$

3. $306037 \mathrm{3E}+02$

3. $3205706 \mathrm{E}+02$

3. $3349151 E+02$

3. 34\%1027E+02

3. $3631436 E+02$

3. $3770435 E+02$

3. $3208028 E+02$

3. $4044207 E+02$

S. $4178775 E+02$

3. $4312372 E+02$

3. $4444403 E+02$

$3.4575135 E+02$

$3.47044 \% 2 E+02$

3. $4932535 E+02$

3. $4959326 E+02$

3. $503430 E E+02$

$3.5206956 \mathrm{E}+02$

3. $5331 \% 30 \mathrm{E}+0$

$3.5453557 E+02$

$3.5574076 \mathrm{E}+02$

$3.5633525+02$

3. $\operatorname{SE} 11412 \mathrm{E}+0 \mathrm{Z}$

$3.5925251 E+02$

$3.6043952 \mathrm{E}+02$

3. $6.159491 \mathrm{E}+02$

$3.6271853 E+02$

3. $6354108 E+02$

3. $6495230 E+02$

$3.6605251 E+02$

$3.6714150 E+02$

3. SE21537E+02

3. $625752 E+02$

3. $7034437 E+02$

3. $7139094 \mathrm{E}+02$

3. $7242734 E+02$

$3.734523 E+02$ 
RHOU

(G/CMB)

.15100

.15200

.15300

.15400

.15500

.15600

.15700

.15300

. 15900

.16000

.16100

.16200

.16300

.16400

- 16500

.16600

.16700

.16800

.16900

.17000

.17100

.17200

.17300

.17400

17500

.17600

.17700

.17300

.17900

.18000

.18100

.15200

$.1830 \%$

. 18400

- 18500

. 18600

- 18700

.15800

.18900

.19000

.15100

.19200

.19300

.19400

.19500

.19600

.19700

.19500

$.19 \% 00$

.20000
C(FHOU, 0.2$)$

(SEC-1)

3. $7446646 E+02$

3. $7547411 \mathrm{E}+02$

$3.76 .46 \% 92 E+02$

$3.774553 \%+02$

$3.7543223 E+02$

3. $793 \% 373 \mathrm{E}+02$

5. $8035595 E+02$

$3.5130 .55 \mathrm{E} E+02$

3. E24197E+02

$3.517137 \mathrm{E}+02$

$3.840 \% 142 E+02$

3. $8500274 E+02$

$3.8590493 \mathrm{E}+02$

3. $8679: 72 \mathrm{E}+02$

3. $5763363 E+02$

3. $6555200 \mathrm{E}+02$

3. $5942777 E+02$

$3.9023707 E+02$

$3.9113350 E+02$

3. $9198129 E+02$

$3.92515 \% 5 E+02$

$3.7364265 E+02$

3. $9446.16 Z E+02$

3. $9527257 E+02$

$3.9607577 E+02$

$3.9637123 \mathrm{E}+02$

$3 . \$ 76.5 \% 24 E+02$

3. $9843 \% 50 E+02$

$3.9721240 E+02$

$3.999732 \mathrm{E}+02$

4. $0073653 E+02$

4. $0143749 \mathrm{E}+02$

4. $0223163 E+02$

4. $029695 \mathrm{E}+02$

4. $056 \% 55 \mathrm{EE}+02$

4. $0442179 E+02$

4. $0513005 E+02$

4. $0534735 E+02$

4. $0655051 E+02$

4. $0724707 E+02$

4. $07736 \mathrm{BEE}+02$

4. $0862035 E+02$

4. $092 \$ 734 E+02$

4. $09 \% 6806 E+02$

4. $1063261 E+02$

4. $1127075 E+02$

4. $1194317 \mathrm{E}+\mathrm{O} 2$

4. $1258725 E+02$

4. $1322934 \mathrm{E}+02$

4. $1386.377 E+02$

A. 10 
RHCU

(G/CMB)

.20100

.20200

.20300

.20400

.20500

.20600

.20700

. 20800

.20500

.21000

.21100

.21200

.21300

.21400

.21500

.21600

.21700

.21500

.21900

.2000

.22100

.22200

.22300

.22400

.22500

.22600

.22700

.22500

.22900

25000

.23100

.23200

$\therefore 3300$

23400

.23500

.23600

.23700

.23300

.23900

24000

.24100

.24200

.24300

.24400

.24500

.24600

.24700

.24800

.24900

.25000
C(FHCII, 0.2$)$

(SEC-1)

4. $1447225 E+02$

4. $15114 \% \mathrm{E}+02$

4. $1573179 E+02$

4. $1634315 E+02$

4. $1694569 E+02$

4. $1754597 E+02$

4. $1 \leqslant 14: 76 . E+02$

4. $187327 \mathrm{E}+02$

4. $1931707 E+02$

4. $19595 E E+02$

4. $2046902 \mathrm{E}+02$

4. $210: 572 \mathrm{EE}+02$

4. $2160055 E+02$

4. $2215025 E+02$

4. $22711 \mathrm{1} \mathrm{EE}+02$

4. $2325930 \mathrm{OE}+02$

4. $235023 \mathrm{EE}+02$

4. $243406.1 E+02$

4. $2457400 E+02$

4. $2540256 E+02$

4. $25.92655 E+02$

4. $26445 B S E+02$

4. $26.96057 E+02$

4. $274706.9 \mathrm{E}+02$

4. $2797632 \mathrm{E}+02$

4. $2547744 E+02$

4. $2557415 E+02$

4. $2746665 E+02$

4. $299546 \mathrm{E}+02$

4. $3043339+02$

4. 3051790E+02

4. $313 \% 35+02$

4. $3186.449 E+0 z$

4. $323315 \mathrm{E}+02$

4. $3279465 E+02$

4. $3325363 E+02$

4. $3370 E 12 Z E+02$

4. $3415759 \mathrm{E}+02$

4. $3460719 E+02$

4. $3505043 E+02$

4. $354 \% 012 \mathrm{E}+02$

4. $35.2595 E+02$

4. $3635905 E+02$

4. $3673600 \mathrm{O}+02$

4. $3721142 E+02$

4. $3763245 E+02$

4. $3805004 E+02$

4. $3646.411 E+02$

4. $38.57466 E+02$

4. $3223176 E+02$ 
RHOU

(G/CMB)

.25100

. 25200

.25300

.25400

. 25500

.25600

.25700

.25000

.25500

.26000

.26100

.26200

.26300

.26400

.26500

.26600

. 26700

.26800

.26900

.27000

.27100

.27200

.27300

.27400

.27500

.27600

.27700

.27800

$.27 \% 00$

.23000

.28100

.23200

.28300

.28400

- 28500

. 28500

. 28700

28800

28700

.29000

29100

.29200

.29300

.29400

.29500

.29600

.29700

. 29800

.29900

.30000
C(RHOU, 0.2)

(SEC-1)

4. $3969535 E+02$

4. $4005570 E+02$

4. $404525 E+02$

4. $40576.15 E+02$

4. $41266.41 \mathrm{E}+02$

4. $416.547 E+02$

4. $4203734 \mathrm{E}+02$

4. $4241795 E+02$

4. $4279543 E+02$

4. $4316957 E+02$

4. $435410 S E+02$

4. $4390929 E+02$

4. 442744BE+O=

4. $4463667 E+02$

4. $4499555 E+02$

4. $4535220 E+02$

4. $457056 E E+02$

4. $460.5612 E+02$

4. $4640363 E+02$

4. $4 \therefore 74580 E+02$

4. $470 \% 057 E+02$

4. $474500 E E+02$

4. $4776.672 E+02$

4. $451006 \mathrm{E} E+02$

4. $484 \Xi 191 E+02$

4. $4976043 E+02$

4. $490 \mathrm{GS} 36 \mathrm{E}+02$

$4.4 \% 40971 E+02$

4. $4973056 E+02$

4. $5004873 E+02$

4. $5036.455 E+02$

4. $5067777 E+02$

4. $509 E \mathrm{ES} \% \mathrm{E}+02$

4. $51296 \% 6 \mathrm{E}+02$

4. $5160239 \mathrm{E}+02$

4. $51906.37 \mathrm{E}+02$

4. $5220757 E+02$

4. $5250645 E+02$

4. $5250274 \mathrm{E}+02$

4. $5.09714 \mathrm{E}+02$

4. $53=3902 E+02$

4. $5307372 E+02$

4. $533662 \mathrm{OE}+02$

4. $542513 \% E+02$

4. $545344 \Leftrightarrow E+02$

4. $54815.5 E E+02$

4. $550 \% 414 E+02$

4. $55370 \Omega 0 E+02$

4. $556.45 .32 E+02$

4. $5571732 E+02$ 
ALULERIS

$$
\begin{aligned}
& M=0.4 \\
& H(C: M)=7.62 \\
& B=0.00000
\end{aligned}
$$

THHLI

(LIC:MS)

C. (FHLII, 0.4$)$

$(E E[-1)$

.01100

E. 24ED006E+01

.01200

5. $642511 E+01$

.01300

E. $13505.2 E+01$

.01400

6. $5705275 E+01$

.01500

$7.001 \% 703 E+01$

.01600

$7.4251979 E+01$

.01700

$7.54 \% 70 E E E+01$

.01800

5.2651

.01000

E. $6797131 E+01$

. 02000

- 0E03130+01

. 02100

$\because 48 \% 41 \Xi 6 E+01$

.02200

\% . $300000 \mathrm{OS}+01$

.02500

1. $02 E 225 E+02$

$.02400^{\circ}$

1. $0672000 E+02$

1. $10575 \% 6 E+02$

1. $1439873 E+02$

1. $2515111 \mathrm{E}+0 \mathrm{2}$

1. $2130 \mathrm{OB} \mathrm{B}+02$

1. $25.57544 \mathrm{E}+02$

1. $2027 \mathrm{E}+02$

1. $20294 \mathrm{iE}+0 \mathrm{Z}$

1. $56.360 \mathrm{GH}+02$

1. $59 \% 416 E+02$

1. $4341: 17 \mathrm{E}+02$

1. $4656357 E+02$

1. 5027625E+02

1. $536119 E+02$

1. $56104 \mathrm{E}+02$

1. $6029200 E+02$

1. $6.355657 E+02$

1. $66.79615 \mathrm{E}+\mathrm{C2}$

1. $6.99075 E+02$

1. $7314024 \mathrm{E}+02$

1. $7626453 E+02$

1. $793554 E+02$

1. $5241256 E+02$

1. $5543600 \mathrm{E}+02$

1. $5042656 E+02$

1. $5135437 E+02$

1. $9431030 E+02$ 
FHOLI

( $G / \mathrm{CM}_{3}$ )

.05100

.05200

. 05500

.05400

. 05500

.05600

.05700

.05800

$.05 \% 00$

- 00000

- 015100

. 00200

.06300

.06400

.06500

.06500

. 06.700

.06500

.06900

.07000

.07100

.07200

.07300

.07400

.07500

.07600

.07700

.07800

.07800

.09000

- 05100

- 05200

.08300

- 05400

- 08500

.05600

.08700

.08800

.05900

. 09000

$.0 \% 100$

. 02200

.08300

$.0 \% 400$

.09500

$.0 \% 600$

.09700

. 09500

$.09 \% 00$

.10000
C: (RHLIUI, 0.4$)$

(SEL:-1)

1. $5720505+02$

$2.0000627 E+02$

2. $0207700+02$

2. $0569856 E+02$

$2.034692 \mathrm{Z} E+02$

$2.1120142 \mathrm{E}+02$

2. $1391 \% 5 E+0$.

2. $1660062 E+02$

Z1925010 19

2. 21078.9E+02

$\therefore 2447165 E+02$

2. $2703030+02$

2. $295907 E+05$

2. $300046+02$

$2.345750 E+02$

2. $970340 E E+0$

2. $546565 E+02$

2. $4157124 E+02$

2. $4425111 \mathrm{E}+0$

2. $4030052 E+02$

2. $4595 E E E+02$

2. $5123 \% 14 \mathrm{E}+02$

2. $5051944 E+02$

$2,557742=525$

$2.5600521 E+02$

$2.60212 \% 1 E+02$

$2.625660 E+02$

2. 64557010E+02

$2,6060424 \mathrm{E}+02$

2. $6100=0 \mathrm{E}+02$

$2.7050172 E+02$

2. $7207140 E+02$

$2.750175 \mathrm{E}+02$

$2.7704607 E+02$

2. $7 \% 0507 \% \mathrm{E}+02$

2. $5103480 \mathrm{E}+02$

2. $5299746 E+02$

$2.349303 E+02$

2. $8 \Leftrightarrow E 6054 E+02$

2. $5157621 \mathrm{EE}+02$

2. $9064332 E+02$

2. $7250474 E+02$

$2.945465 E E+02$

$2.9610060 \mathrm{O}+02$

2. $9797230+02$

$2.975652 E+02$

G $01526 E+02$

$3.032637 E+02$

․ $04 \% 945 E+02$

$3.0670 \% 10 E+02$

A. 14 
FHOLI

(G/CMB)

.10100

.10200

.10300

.10400

.10500

.10000

.10700

- 10500

$.10 \% 00$

.11000

.11100

.11200

.11500

.11400

.11500

.11600

.11700

$.11800^{\circ}$

.11900

- 12000

.12100

.12200

- 12300

.12400

.12500

.12800

.12700

. 12800

.12900

, 13000

. 13100

- 13200

.13300

.13400

.13500

.13600

.13700

. 13800

. 13000

.18000

.14100

.14200

.14300

.14400

.14500

.14000

.14700

.14300

$.14 \% 00$

.15000
C: (RHLIU, 0.4$)$

(SEC:-1)

$3.0540190 E+02$

$\because 1007736 E+02$

S. $117357 E+02$

S. $133757 \%+02$

- $14 \% 97 \%$

3. $1600636+02$

Э. $1 \mathrm{E} 1 \% 5 \mathrm{Z} E+02$

$3.1976 .960 \mathrm{E}+02$

$3.213657 E+02$

3. $220672 \mathrm{E}+02$

$3.243926 \mathrm{E}+02$

3. $250206 \mathrm{O}+02$

$3.273 \% 594+02$

3. 20:7461E+02

3 . $5030740 E+02$

3. $317 \mathrm{E} 614 \mathrm{E}+02$

3. 3引E1\% $2 E+02$

3. $3463747 E+02$

S. $3604174 E+02$

$3 . \$ 74310 \mathrm{E}+0 \mathrm{Z}$

3.

$3.4016795 E+02$

$3.4151550 E+02$

$3.4294 \% 00 \mathrm{E}+02$

$3.4416 \% 1 E E+02$

$3.454754 \mathrm{E}+02$

$3.46 .76 \% 1 \mathrm{EE}+02$

$3.4904 \% 47 E+02$

$3.4931604 \mathrm{E}+02$

3. 50.57141E+02

3. $5151302 E+02$

3. $5.304211 E+02$

3. $54259 \% \mathrm{E}+02$

3. $5.546060+02$

3. $5665.567 \mathrm{E}+\mathrm{C} 2$

3. $57: 0613 E+02$

$3.500454 E+02$

3. $0010117 E+02$

3. $6.130644 E+0=$

$3.64401 \% E+02$

3. $550,261 E+02$

ت. $646736 \mathrm{E}+0$

$3.57735 \%+02$

S. GCEKI57E+02

$3.6 .7 \% 4077 E+02$

Z. $600001 \mathrm{E}+02$

$2.700652 \mathrm{E}+02$

․ $7111175 E+0$

$3.7214779 \mathrm{E}+02$

$3.7317374 E+02$ 


\section{FHOU \\ (G/CM3)}

.15100

.15200

. 15300

- 15400

- $15500^{\circ}$

.15600

.15700

.15800

.15800

.16000

- 16100

.16200

- 16300

.16400

.16500

.16000

.16700

. 15800

.16900

.17000

.17100

.17200

.17300

.17400

.17500

.17600

.17700

.17800

.17900

.19000

- 15100

- 18200

.13500

.18400

- 15500

.18600

.18700

- 158000

- 19500

.19000

.19100

.19200

.19500

.17400

.19500

.19600

.19700

.19800

$.19 \% 00$

- 20000
C(RHOU, 0.4 )

(SEC-1)

$3.741892 \mathrm{EE}+0$ $3.751 \% 479 E+02$ $3.7819057 E+0=$ 3.771765 CE+02 3.7E15277E+02 $3.7 \% 1192 \mathrm{VE}+0$ S. $8007651 E+02$ 3. $9102426 \mathrm{a}+02$ 3. $319200 \mathrm{O}+02$ 3. B2E $16 E+02$ 3. E $35127 \mathrm{C}+0$ $\therefore 54726 \mathrm{E}+12$ 2. $5562614 \mathrm{E}+02$ $3.60,1900 E+02$ $3.5740455 E+02$ 3. $E: 25102 E+02$ $3.5914902 E+12$ $3.9000364 E+02$ $3.90 E 5991 \mathrm{E}+0 \mathrm{Z}$ $3.71702 \% 5 E+02$ $3.925374 \mathrm{E}+02$ 3. $336475 E+02$ $3.941 E 34 E+02$ 3. 9 त. $487 E+02$ $3.95 \% 9616 E+02$ $3.9659561 E+02$ $3.97351=7 E+02$ 2. $9316 \mathrm{EE}+02$ $3.96 \% 55 E E+02$ $3.9 .770152 \mathrm{E}+02$ 4. $00459 \mathrm{BEE}+02$ 4. O121127E+0 $4.01 \% 5556+12$ 4. $026.272 E+02$ 4. $0342303 E+02$ $4.04146 .41 E+0)$ 4. $040625 E=02$ 4. $05572705+02$ 4. $06275=0$ 4. $0607257 E+02$ 4. $07 \Leftrightarrow 20-02$ 4. $0534 \leq-0 E+02$ 4. $0702060 \mathrm{E}+\mathrm{O} 2$ 4. $0 \% 6452 E+02$ 4. $1035932 E+02$ 4. $1101737 E+0$. 4. $1167041 E+02$ 4. $1231670 E+02$ 4. $1295734 \mathrm{E}+02$ 4. $135196+02$ 


\section{FHOU}

(G/CM3)

.20100

.20200

. 20300

.20400

. 20500

. 20600

. 20700

.20500

.20900

. 21000

. 21100

21200

21300

21400

21500

.21600

.21700

.21800

.21500

- 2000

.22100

.22200

.22300

.22400

- 22500

.22600

- 22700

.22800

.22900

.25000

.25100

. 23200

.23300

.23400

.23500

.23600

.23700

. 23000

.23900

.24000

.24100

.24200

.24300

.24400

.24500

.24600

.24700

.24800

.21500

.25000
C: (FHCIUI, 0.4$)$

(SEC-1)

4. $1422076 E+02$

4. $1454: 76 . E+02$

4. $15.46057 E+02$

4. $1607247 E+02$

4. $1667050 E+02$

$4.172701 E+02$

4. $1797410 \mathrm{O}+\mathrm{OL}$

4. $134 \therefore 3670+02$

4. $15047 \% 4 E+02$

4. $196209 E+02$

4. $2020050 E+02$

4. $2076011 E+02$

4. $2130 \mathrm{E}+02$

4. $2190 \mathrm{OSE}+02$

4. $24440 E E+02$

4. $2205 \mathrm{O}=2$

4. $235557 \mathrm{E}+02$

4. $2407445 E+02$

4. $2460 E 2 \mathrm{E}+02$

4. $251: 720 E+02$

4. $2560151 E+02$

4. $2015122 E+02$

4. $26.656 .1 \mathrm{E}+02$

4. $2720675 E+02$

4. $2771290 E+02$

4. $281424 E+02$

4. $\because 1130 E+02$

4. $2020400 E+02$

4. $2 \% \Leftrightarrow 2 \% E+02$

4. $30176 G C E+02$

4. $30 \leqslant 565 E+02$

4. $311323 E+02$

4. $31603 \% 6 E+02$

4. $3207132 E+02$

4. $3253467 E+02$

4. $32,9421 E+02$

4. $3344962 E+02$

4. $33 \% 0124 E+02$

4. $34: 438 \% E+02$

4. $347 \% 25 E+02$

4. $352359 E+02$

4. $3566055 E+02$

4. $5610127 E+02$

4. $3653015 E+02$

4. $36 \% 55=34+02$

4. $37376.57+2$

4. $377 \% 4 \% 4 E+02$

4. $32004 E+02$

4. $3002029 E+02$

4. $3702776 E+02$ 
RHOL

( $\mathrm{G} / \mathrm{CM} 3$ )

25100

.25200

25300

. 25400

- 25500

- 25600

.25700

- 25800

.25900

.26000

- 26100

.26200

. 26300

.26400

.26500

.26600

.26700

. 20680

$.26 \% 00$

27000

27100

27200

27300

27400

27500

.27600

. 27700

.27500

27900

- 28000

28100

- 28200

.28300

.28400

- 28500

- 28600

.28700

- 28500

- 28900

.29000

.29100

.29200

.29300

.27400

.24500

.27600

.29700

. 28300

. 29900

. 30000
C(RHOU, 0.4$)$

(SEC-1)

4. $3943131 E+02$

4. $32532505+02$

4. $402 z=E E+02$

4. $4062 \exists 7 \overline{7} E+02$

$4.4101460 E+02$

4. $4140196 \mathrm{E}+02$

4. $417 E 621 E+02$

4. $4216716.0+02$

4. $4254518 E+02$

4. $42 \geqslant 1991 E+02$

4. $43215 \mathrm{SEE}+02$

4. $4360034 E+0=$

4. $440257 \% E+02$

4. $4438550 E+0$

4. $4474515 E+02$

4. $4510477 E+07$

$4.454586-1 E+2$

4. $4580 \% 5 E+0$

4. $4615771 E+02$

4. $40.50303 E+02$

4. $46.4552 \mathrm{E}+02$

4. $471552 E+02$

4. $4752 \mathrm{EE}+02$

4. $4755657 E+02$

4. $4 \mathrm{E} 1 \mathrm{BS} 21 \mathrm{E}+02$

4. $4551721 E+02$

4. $4354363 E+02$

4. $4 \% 1 S 732 E+02$

4. $4948554 E+02$

4. $4980730 E+02$

4. $5012345 E+02$

4. $5043711 \mathrm{E}+02$

4. $5074336 E+02$

4. $5105707 E+02$

4. $5136346 E+02$

4. $5166730 \mathrm{E}+02$

4. $5196596 E+02$

4. $5226021 E+02$

4. $5251517 E+02$

4. $5255951 \mathrm{E}+02$

4. $5315212 E+02$

$4.5344224 E+02$

4. $5373011 \mathrm{E}+02$

4. $54015: 0 E+02$

4. $5429 \% 29 \mathrm{E}+02$

4. $545305 \mathrm{BE}+02$

4. $5495982 E+02$

4. $5513685 E+02$

4. $55411 \mathrm{SGE}+02$

$4.556 .847 \mathrm{E}+02$

A. 18 
AQULEULS

$$
\begin{aligned}
& M=0 . E \\
& H(C: M)=7.62 \\
& B=0.00000
\end{aligned}
$$

RiH[it]

(E/LME)

.01100

.01200

.01300

.01400

.01500

.01600

.01700

.01800

.01700

. 02000

.02100

. 02200

.02300

.02400

. 02500

- 02600

. 02700

- 12500

.02900

. 03000

.03100

- 03200

. 03300

.03400

. (13500)

.03600

.03700

- 03000

.03900

.04000

.04100

.04200

.04300

.04400

.04500

.04600

.04700

. 04800

$.04 \% 00$

.05000
E.(FHIIU, O.E)

( $S E E_{-}-1$ )

5. $2374026 E+01$

5. $6322558 E+01$

6. $1221353 E+01$

6. $557146.2 E+01$

6. $787 \equiv 175 E+01$

7. $4126072 \mathrm{E}+01$

7. $533552 E+01$

ง. $243294 E+01$

E.6607124E+01

9. $0675: 342 E+01$

$\$ .4655257 E+01$

. $3076345 E+01$

1. O201140E

1. $0650220 E+02$

1. $1035025 \mathrm{E}+02$

1. $1415550 \mathrm{OE}+02$

1. $17 \% 1 \mathrm{~g} \% \mathrm{E}+02$

1. $2164095 E+02$

1. $2532162 \mathrm{E}+02$

1. $2 \mathrm{EQCO} 19 \mathrm{SE}+\mathrm{OL}$

1. $325620 E+02$

1. $3612256 \mathrm{E}+02$

1. $3964.396 \mathrm{E}+02$

1. $4312641 \mathrm{E}+02$

1. $46.570 E B E+02$

1. $4997740 \mathrm{E}+02$

1. $5334635 E+02$

1. $5600060 \mathrm{E}+02$

1. $5997527 E+02$

1. $6323512 \mathrm{E}+02$

1. $6.645904 E+02$

$1.69647 \% 5 E+02$

1. $7280138 E+02$

1. $75 \% 2105 E+02$

1. $7500655 E+02$

1. $8205370 \mathrm{OE}+02$

1. $8507692 E+02$

1. $E: E 0) 237 E+0 Z$

$1.9101552 E+02$

1. $9393655 E+02$ 
RH[II]
(G/LMS)

.05100

.05200

.05300

. 05400

. 05500

.05600

. 05700

.05500

- 05900

.06000

.06100

.06200

. 06.500

.06400

.06500

- 00000

.06700

. 06800

. 05500

.07000

.07100

.07200

.07300

.07400

.07500

- 07600

.07700

.07800

.07700

.08000

. 05100

.08200

. 08300

.08400

. 0S500

. OEGon

. 03700

- 05800

.08700

. 09000

. 0\$10()

.09200

.09500

.07400

- 0\$500

. 0800

.09700

.09500

.08700

.10000
C. (FIHL̈LI, O.\&)

(SEL:-1)

$1.9682586 E+02$

1. $\$ 963400 E+02$

2. 0:5111 $\mathrm{EE}+02$

$2.0530704 E+02$

2. $0907258 E+02$

2. $1080578 E+02$

2. 1351 5ESE+02

2. $161933 \mathrm{GE}+02$

2. $1834145 E+02$

2. $2146111 \mathrm{E}+02$

$2.2405202 E+02$

$2.2601595 E+02$

2. $2 \$ 15157 E+02$

2. $3166034 \mathrm{E}+02$

$2.3414075 E+02$

2. $3059564 E+02$

2. $3902509 E+02$

2. $4142741 E+C 2$

$2.4380417 E+02$

$2.4615571 E+02$

2. $4848161 \mathrm{E}+02$

2. $5078286 . E+02$

2. $590596 \mathrm{GE}+02$

$2.5531147 E+02$

$2.5753993 \mathrm{E}+02$

$2.5974461 E+02$

$2.6192569 E+02$

$2.640840 \mathrm{EE}+02$

$2.5621576 E+02$

$2.6333043 E+02$

$2.7042014 \mathrm{E}+02$

$2.7248 E 1 E E+02$

$2.745322 \mathrm{E}+02$

2. $7655776 E+02$

$2.735606 .8 E+0=$

2. $9054232 E+02$

2. $8250201 \mathrm{E}+02$

2. $8444220 E+02$

2. $86361 E_{1} \mathrm{E}+02$

2. BU20103E+02

$2.901401 E E+02$

$2.9200001 \mathrm{E}+02$

$2.9903937 E+02$

2. $950600 \mathrm{E}+02$

$2.9746152 E+02$

2. $924447 E+02$

3. $0100515 \mathrm{E}+02$

3. $0275394 E+02$

3. 04490\%\$E+0z

$3.0619037 E+02$ 
FH[II]

( $\left[1 / M_{3}\right.$ )

.10100

.10200

.10300

.10400

.10500

.10600

.10700

.10800

.10500

.11000

.11100

.11200

.11500

.11400

.11500

.11600

.11700

.11800

.11900

.12000

.12100

- 12200

.12300

.12400

.12500

.12600

.12700

- 12000

.12500

.13000

. 13100

.13200

. 13300

.13400

.13500

. 13600

. 13700

.13300

.13900

.14000

.14100

.14200

.14500

.14400

.14500

.14600

.14700

.14500

.14500

.15000
C. (FIHLILI, $\left.0 . E^{2}\right)$

( $E E[-1)$

$3.0780210 \mathrm{E}+0$

3. $0755547 E+02$

S. $11211 \% \bar{E}+02$

$3.1235126 \mathrm{E}+02$

3. $1447371 \mathrm{E}+02$

$3.1607091 \mathrm{E}+02$

$3.176674 \mathrm{E}+02$

3. $19235 \mathrm{EE}+02$

3. $207 \% 602 E+02$

3. 2233547E+02

3. $2365,942 E+02$

3. $25367 \% 1 \mathrm{E}+02$

3. 26E60076E+02

․ $2830345 E+02$

3. $2850035+02$

$\because 3124751 E+02$

3. $326.7971[+02$

3. $3409745 E+02$

$3.3550052 E+02$

3. 36.8E. $61 E+02$

3. $3820400 \mathrm{O}+02$

3. $396262 E+02$

3. $409715 \mathrm{E} E+02$

3. $423041 \% E+02$

G. $45205 E+02$

$3.44 \% 2 \% 74 E+02$

3. $462221 \mathrm{E}+02$

$3.4750214 E+02$

3. $45765 E B E+02$

3. $5002292 E+02$

$3.5126592 E+02$

․ $524201 E+02$

3. $5370917 E+02$

3. $54 \% 12 E E E+02$

$3.5610475 E+02$

$3.5723457 E+02$

Э. 5.4.5 $315 E+02$

$3.5 \% 60.4 \mathrm{E}+02$

$3.6075424 E+02$

3. $61 \mathrm{E}: 794 \mathrm{E}+02$

3. 6.300\%9EE+02

$3.6412067 E+02$

S. $652006 E+02$

$3.663003 E E+02$

$3.6 .730719 E+02$

$3.63454605+02$

$3.6751142 E+02$

$3.7055700 E+02$

3. $715 \% 3 E+02$

$3.7201967 E+02$ 
RHCIUI

(E/LMB)

.15100

.15200

.15500

.15400

.15500

.15600

.15700

.15800

.15900

.16000

.16100

.16200

.16300

.16400

.16500

.10600

.16 .700

.16500

.16900

.17000

.17100

.17200

.17300

.17400

.17500

.17600

.17700

.17800

$.17 \% 00$

.18000

.18100

.18200

.18300

.18400

.18500

.18600

.18700

. 18800

.18900

.17000

.19100

.19200

.19300

.19400

.19500

.19600

.19700

.19800

.19900

.20000
C: (FIHCIU, 0.8$)$

(SEL-1)

$3.736349 \% E+02$

3. $740404 E E+02$

3.7565642E+02

3. $762222=0+02$

3. $7759836 E+02$

S. 7356515E+OL

3. $7952229 E+0=$

$3.30470 \times B E+02$

3. $8140373 E+02$

3. $3233524 E+02$

$3.5325 E \Omega 3 E+02$

$3.5416986 \mathrm{E}+02$

3. $550726 \% \mathrm{E}+02$

$3.85 \% 6033 E+02$

3. E6ES1 $52 E+02$

$3.3772317 E+02$

3. $6 E 55622 E+02$

S. $3945605 E+02$

$3.905076 \quad 12$

$3.9115057 \mathrm{~L} \mathrm{~N}$

$3 . \$ 195601 E+02$

3. $2231322 E+02$

$3.9363272 E+02$

$3.944437 \mathrm{EE}+02$

$3.8524749 E+02$

$3.9604357 E+02$

$3.90631 \mathrm{EE}+02$

3. $9761206 E+02$

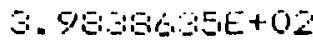

$3.9715250+02$

$3.95 \% 1164 \mathrm{E}+02$

4. $0060334 E+02$

4. $01407 \% 2 E+02$

4. $0214506 E+02$

4. $0257621 E+02$

4. $0.30014 \mathrm{E}+02$

4. $0431703 E+02$

4. $050274 \mathrm{EE}+02$

4. $0573101 E+02$

4. $0042012 E+02$

$4.071156 .4 E+02$

4. $0750260 E+02$

4. $0.94906 .3 E+02$

4. $0 \% 15210 E+02$

4. $0 \% \Omega 1742 E+02$

$4.10476 .4 \mathrm{EE}+02$

$\therefore 111295 \mathrm{~B}+02$

4. $11776.600+02$

4. $124175 \mathrm{OUE}+\mathrm{OZ}$

4. $1505270 E+02$ 
FHCIII

( $G / C M B)$
[. (FHLULI, 0.8 )

$(S E E-1)$

\begin{tabular}{|c|c|}
\hline $\begin{array}{l}.20100 \\
.20200\end{array}$ & $\begin{array}{l}\text { 4. } 136 E 217 E+02 \\
\text { 4. } 1430565 E+02\end{array}$ \\
\hline .20300 & 4. $1492=46 E+02$ \\
\hline .20400 & 4. $1553564 E+02$ \\
\hline .20500 & $4.16 .1428 \mathrm{EE}+0$ \\
\hline .20000 & $4.1674334 E+02$ \\
\hline .20700 & 4. 1733G97E+0Z \\
\hline .20000 & $4.179 .91 E+02$ \\
\hline .20900 & 4. $1951415 E+O 2$ \\
\hline .21000 & $4.190 \% 375 E+02$ \\
\hline .21100 & $4.1 \% 66005 E+02$ \\
\hline .21200 & 4. $202=72 B E+02$ \\
\hline .21300 & 4. $20 E 0140 E+02$ \\
\hline .21400 & $4.213603 E E+02$ \\
\hline .21500 & 4. $2191431 \mathrm{E}+02$ \\
\hline .21600 & $4.22463=2 E+02$ \\
\hline .21700 & 4. $2300745 E+02$ \\
\hline 21500 & 4. $254607 E+02$ \\
\hline .21500 & 4. $240 E: 101 E+02$ \\
\hline .22000 & 4. $2761074 E+02$ \\
\hline .22100 & $4.2513560 E+02$ \\
\hline .2200 & 4. 256 $523 E+02$ \\
\hline .22500 & 4. $2017153 E+02$ \\
\hline .22400 & 4. 2665320E+02 \\
\hline .22500 & 4. 271 E9EZE+O2 \\
\hline .22600 & $4.27 \theta_{2} \cup 1 E+02$ \\
\hline .22700 & 4. 2E:1 E9E9E+O2 \\
\hline .22800 & 4. 2BC.83G6E+02 \\
\hline .22900 & $4.2917247 E+0.2$ \\
\hline .23000 & $4.2965734 E+02$ \\
\hline .3100 & $4.3013003 E+02$ \\
\hline .23200 & $4.306 .144 \mathrm{EE}+02$ \\
\hline .23300 & $4.3105679 E+02$ \\
\hline .23400 & $4.3155506 E+02$ \\
\hline .23500 & $4.3201924 E+02$ \\
\hline .23600 & 4. $3247941 E+02$ \\
\hline .23700 & $4.3293564 E+02$ \\
\hline 23800 & $4.3335793 E+02$ \\
\hline .2900 & 4. $338: 36.34 E+02$ \\
\hline .24000 & $4.34250 \% 4 \mathrm{E}+02$ \\
\hline .24100 & 4. $347216: 5 E+02$ \\
\hline .242010 & 4. $2515.362 E+02$ \\
\hline .24300 & 4. 355. $\% 204 \mathrm{E}+02$ \\
\hline .24400 & 4. $3602164 E+02$ \\
\hline .24500 & $4.3644753 E+02$ \\
\hline .24600 & 4. $3667000 E+02$ \\
\hline .24700 & 4. 3726673E+02 \\
\hline $.24: 00$ & $4.3770392 E+02$ \\
\hline .24900 & 4. उE11564E+0Z \\
\hline 25000 & $4.3352332 E+0$ \\
\hline
\end{tabular}


FHCIU

(E/LM3)

25100

25200

.25300

.25400

.25500

- 25600

25700

25800

.25900

.26000

.26100

.26200

.26300

. 26400

.26500

.26600

.26 .700

.26800

.26900

.27000

27100

27200

.27300

.27400

.27500

.27000

.27700

.27800

27900

25000

. 28100

. 28200

. 28300

.28400

. 28500

.28600

.28700

. 28800

28900

29000

28100

27200

. 24500

29400

25500

.29600

.25700

. 29800

.29900

.30000
C: (FIHGUU, O.E)

( $S E E:-1$ )

4. 3E92070E+02

4. $393302 \mathrm{EE}+02$

$4.3972540 \mathrm{E}+02$

4. $4012321 \mathrm{E}+02$

4. $4051476 E+02$

4. $4090296 E+02$

4. $4128301 E+02$

4. $416698 \mathrm{E}+02$

4. $4204 E 57 \mathrm{O}+02$

4. $4242411 E+02$

4. $.227 \exists 6.6 \mathrm{E}+02$

4. $43: 6312 E+02$

4. $4353259 E+02$

4. $4389606 E+02$

$4.44256 .57 E+02$

4. $4461399 E+02$

4. $4476.607 E+02$

4. $4532039 E+02$

4. $456.693 B E+02$

4. $4601548 E+02$

4. $4635885 E+02$

4. $4669744 E+02$

$4.4703721 E+02$

4. $4737242 E+02$

4. $47704 \% 2 E+02$

4. $4803467 E+02$

4. $4336.1 \% 4 E+02$

4. $4565657 E+02$

4. $4 \% 0055 E E+02$

4. $492514 E+02$

4. $456450 \%+02$

4. $4 \% 5565 E+02$

4. $502716.9 E+02$

4. $505812 B E+02$

4. 50 OES5:2E+02

4. $5119322 E+02$

4. $514 \% 56.5 E+02$

4. $517.7571 \mathrm{E}+02$

4. $520 \% 34 \% E+02$

4. $523 B 8 \% E E+02$

4. $5269220 E+02$

4. $5297316 . E+02$

4. 5.32․ $134 E+02$

4. 5354 GSEE+02

4. 5GEG272E+02

4. $5411400 E+02$

4. $5437457 E+02$

4. $54 \therefore 7277 E+02$

4. $5.74857 E+02$

4. $5522231 E+02$ 


\section{SOURCE LISTINGS OF DATA ANALYSIS AND OIJTPUT SUBROUTINES}

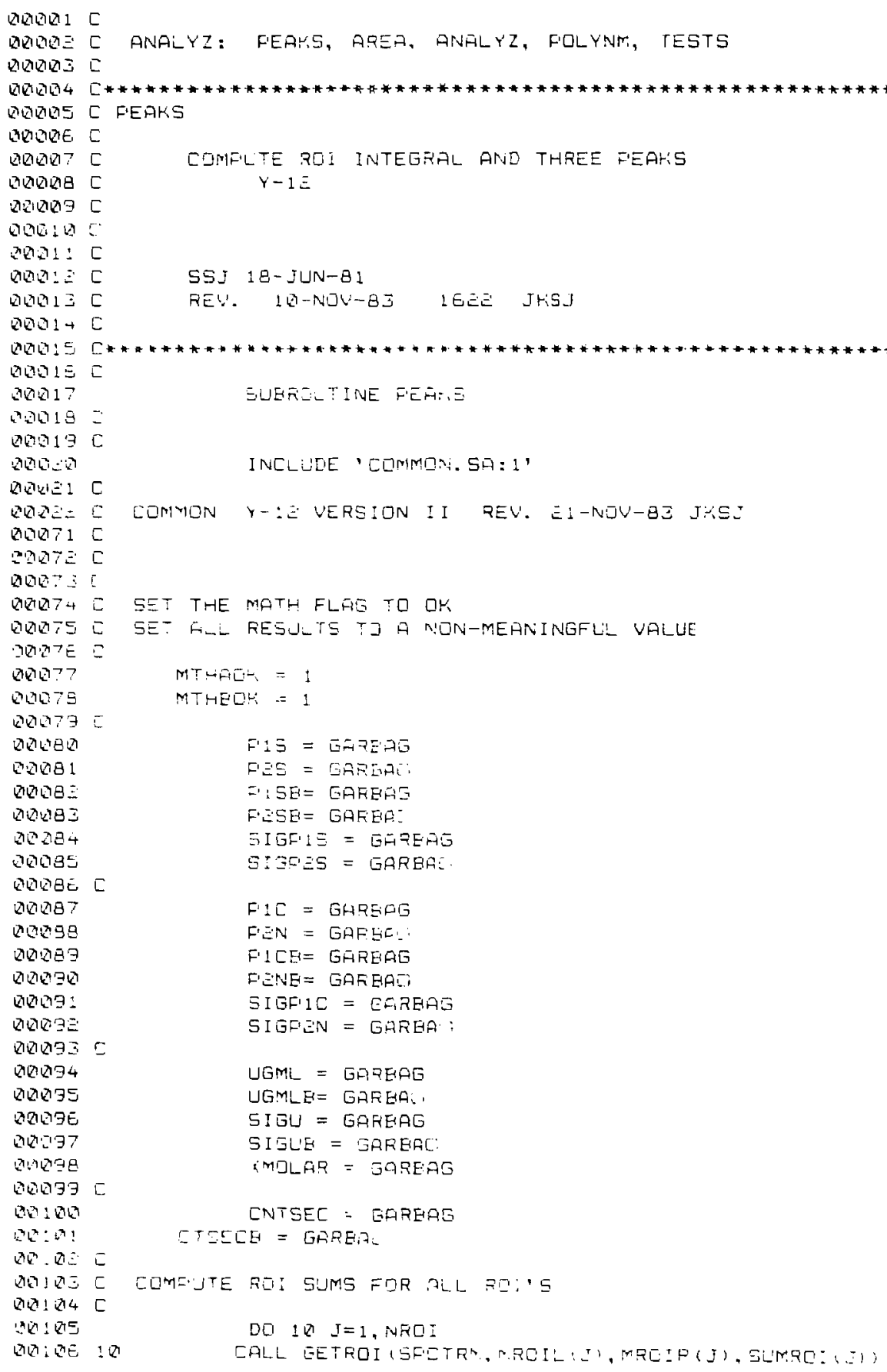




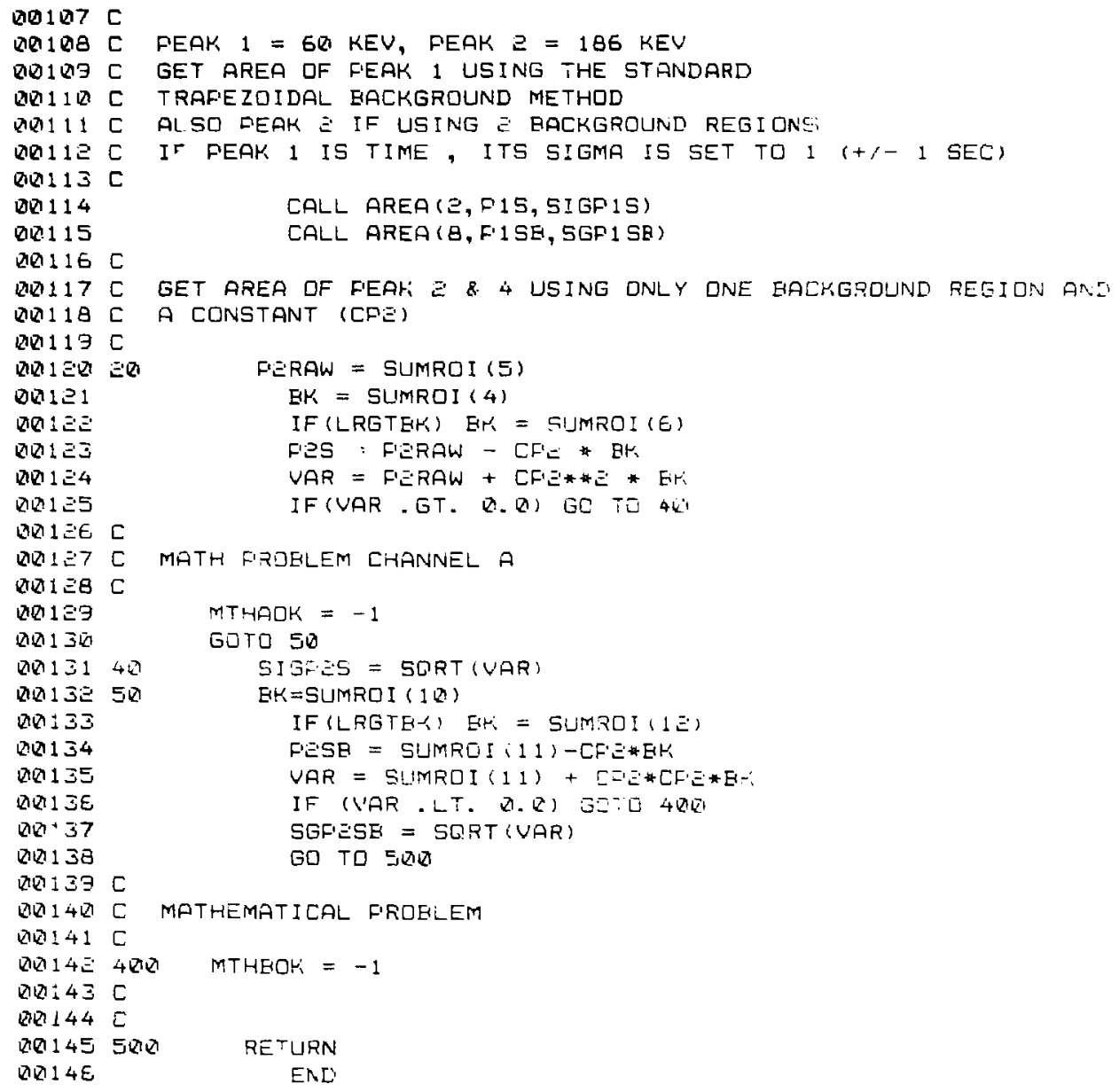




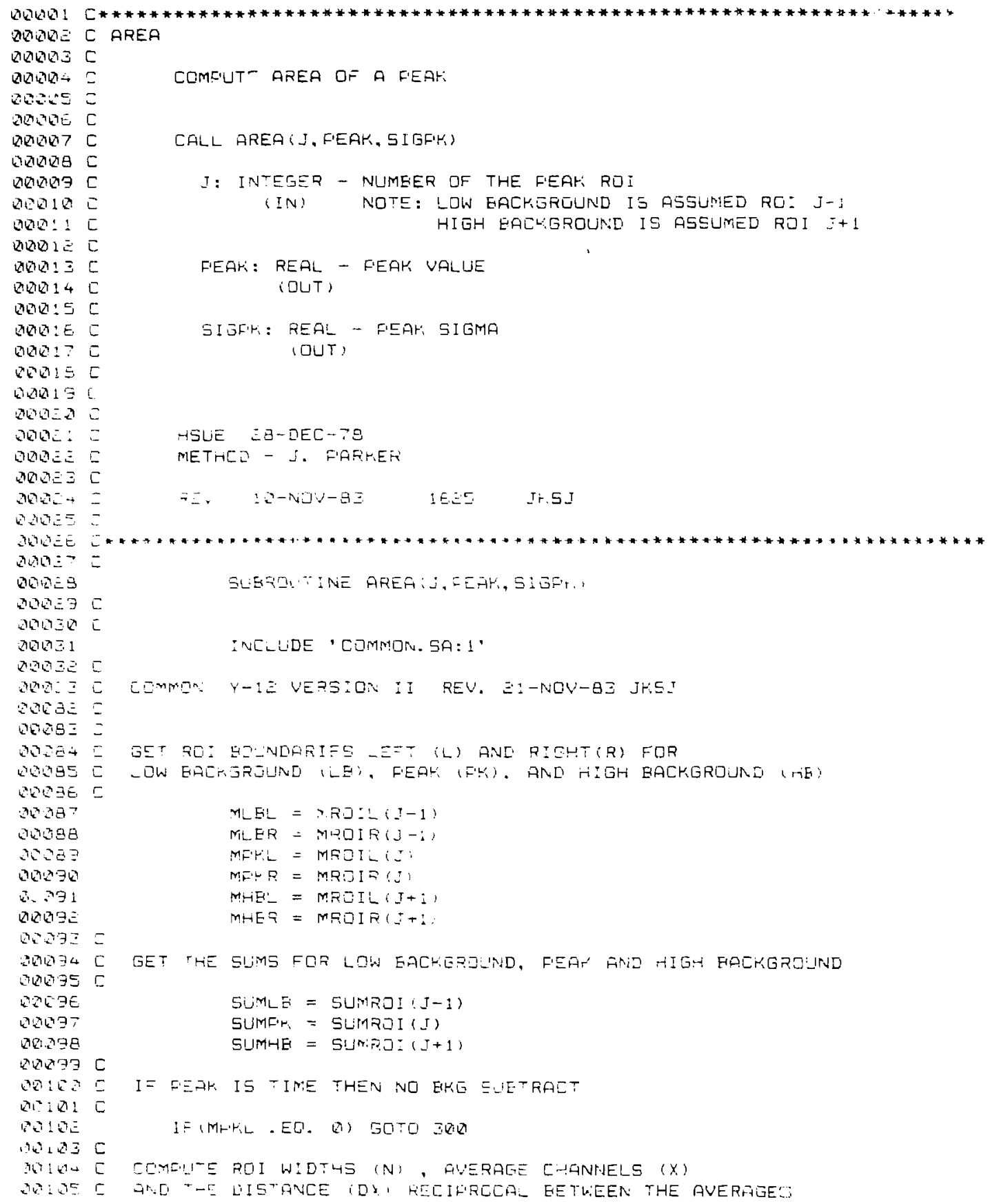




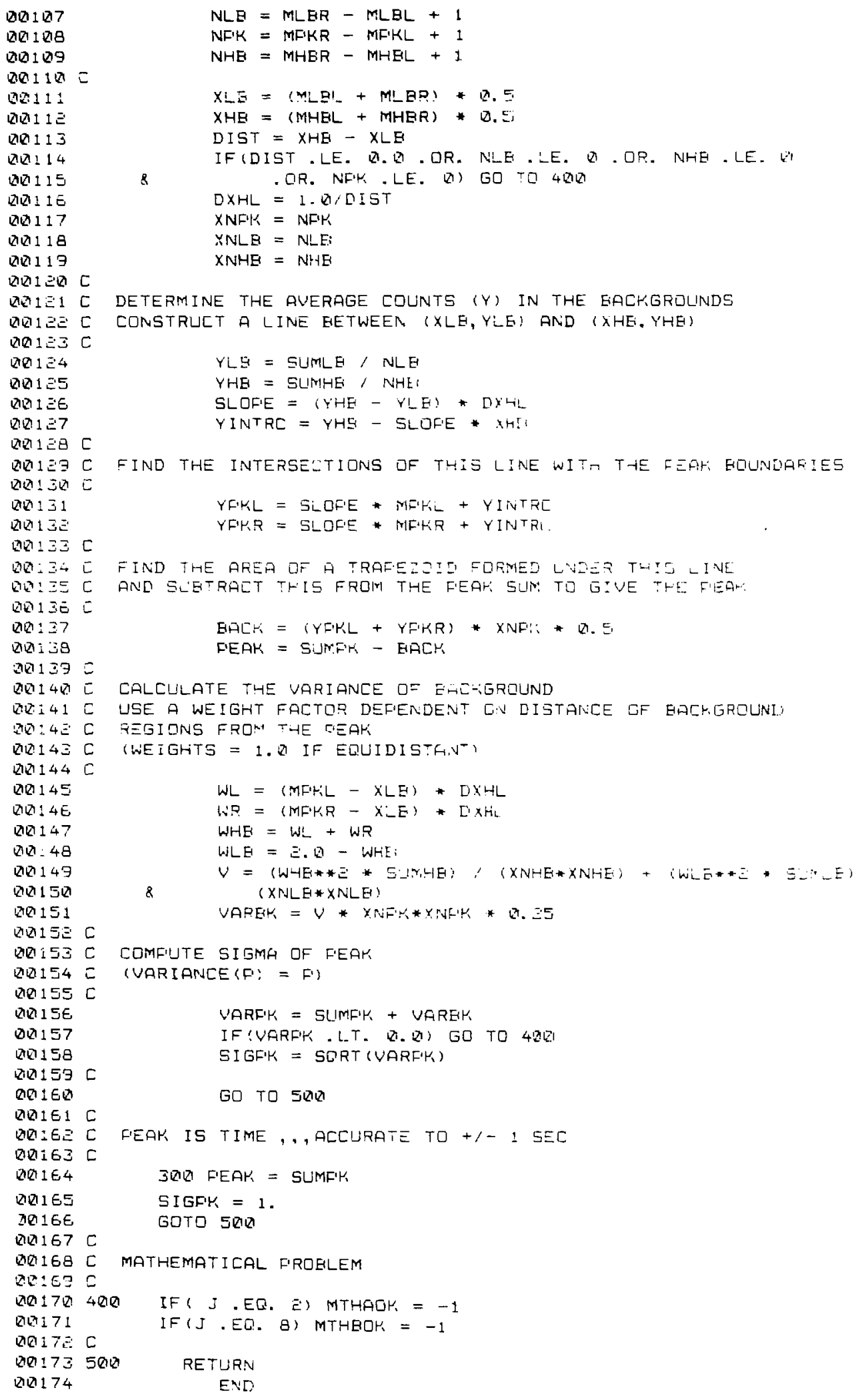




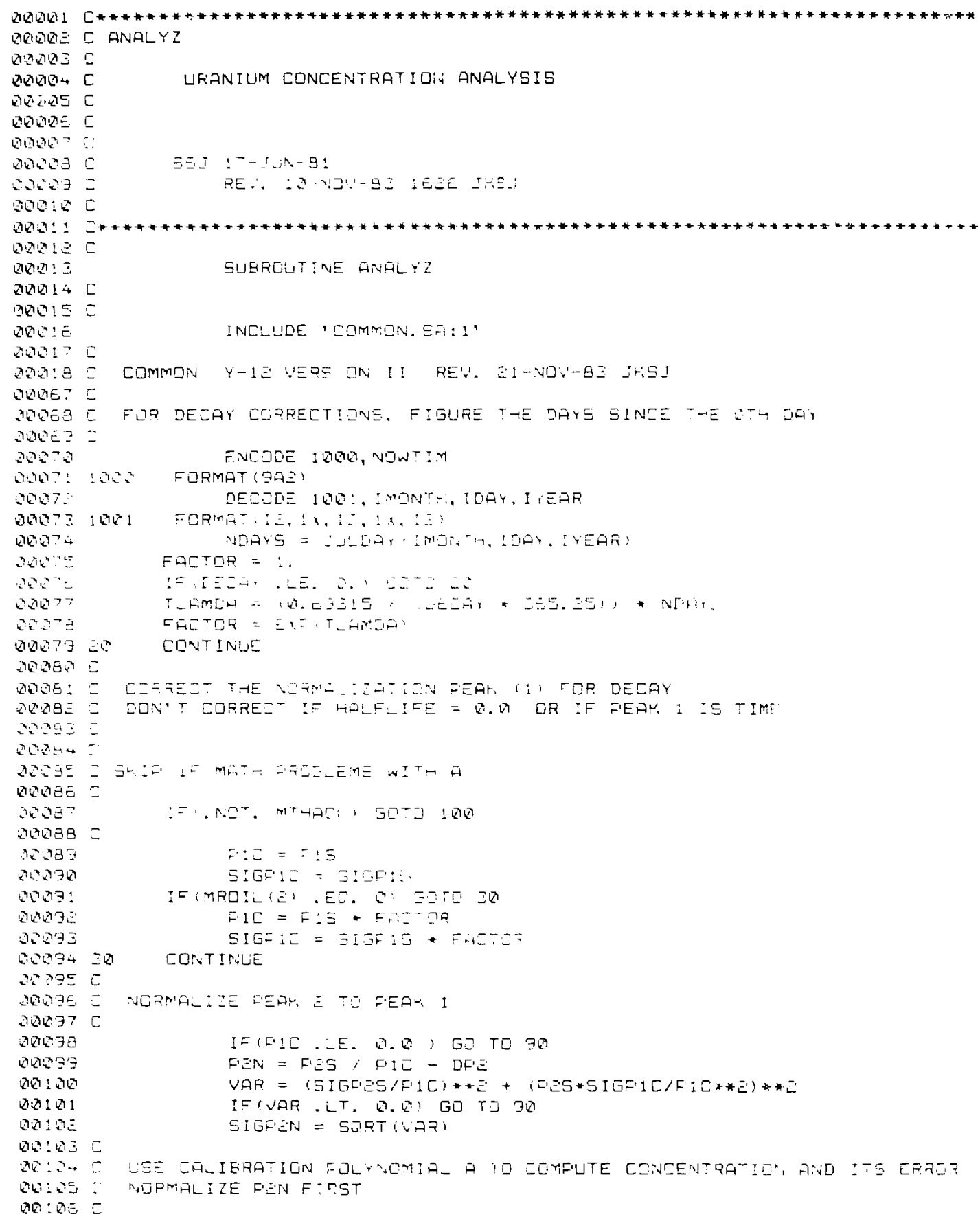




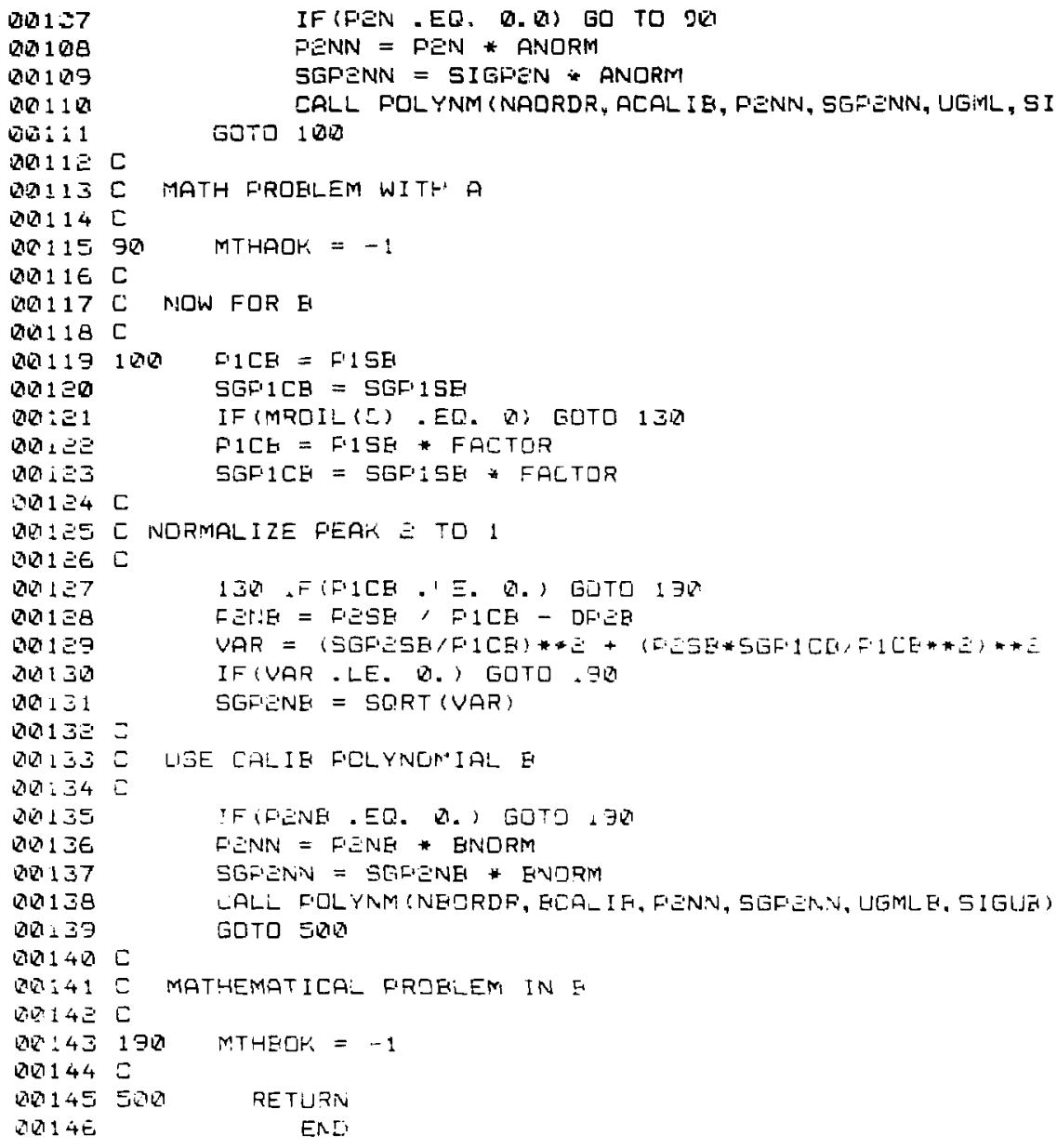


Dovon $1 \mathrm{C}$

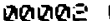

andas

काजिए 4

Dongs 5

Rugue $C$

Q121010 7

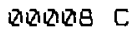

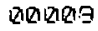

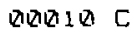

सार्या 1

$\operatorname{\theta in} 20$

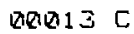

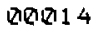

$\operatorname{ana15}$

$\operatorname{\theta in} 16$

परिख 17

काष 18

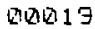

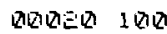

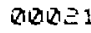

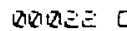

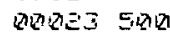

Qराप्ति

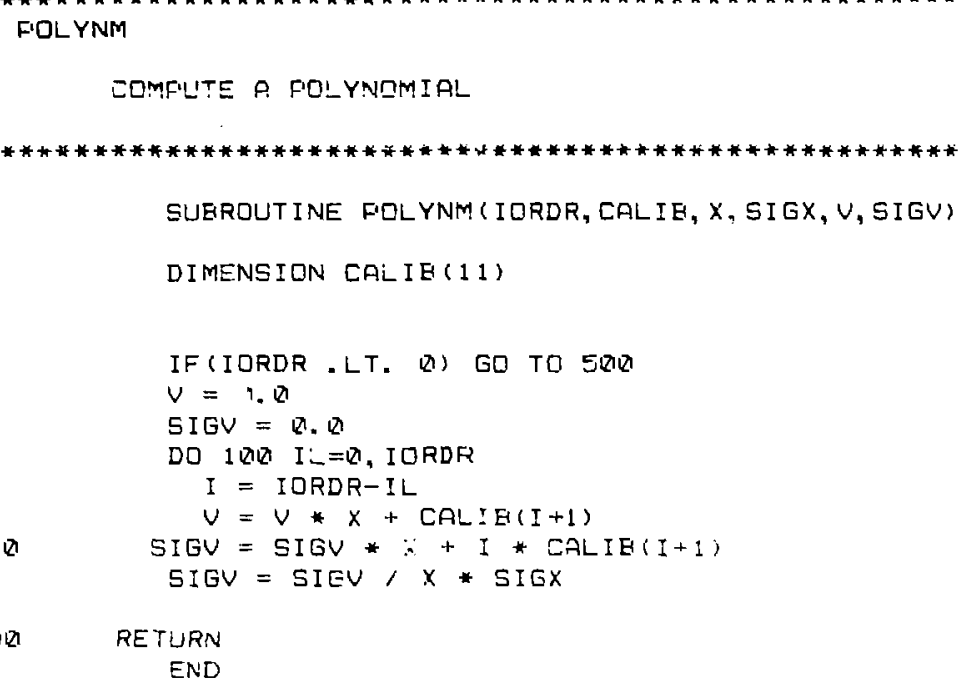

END 


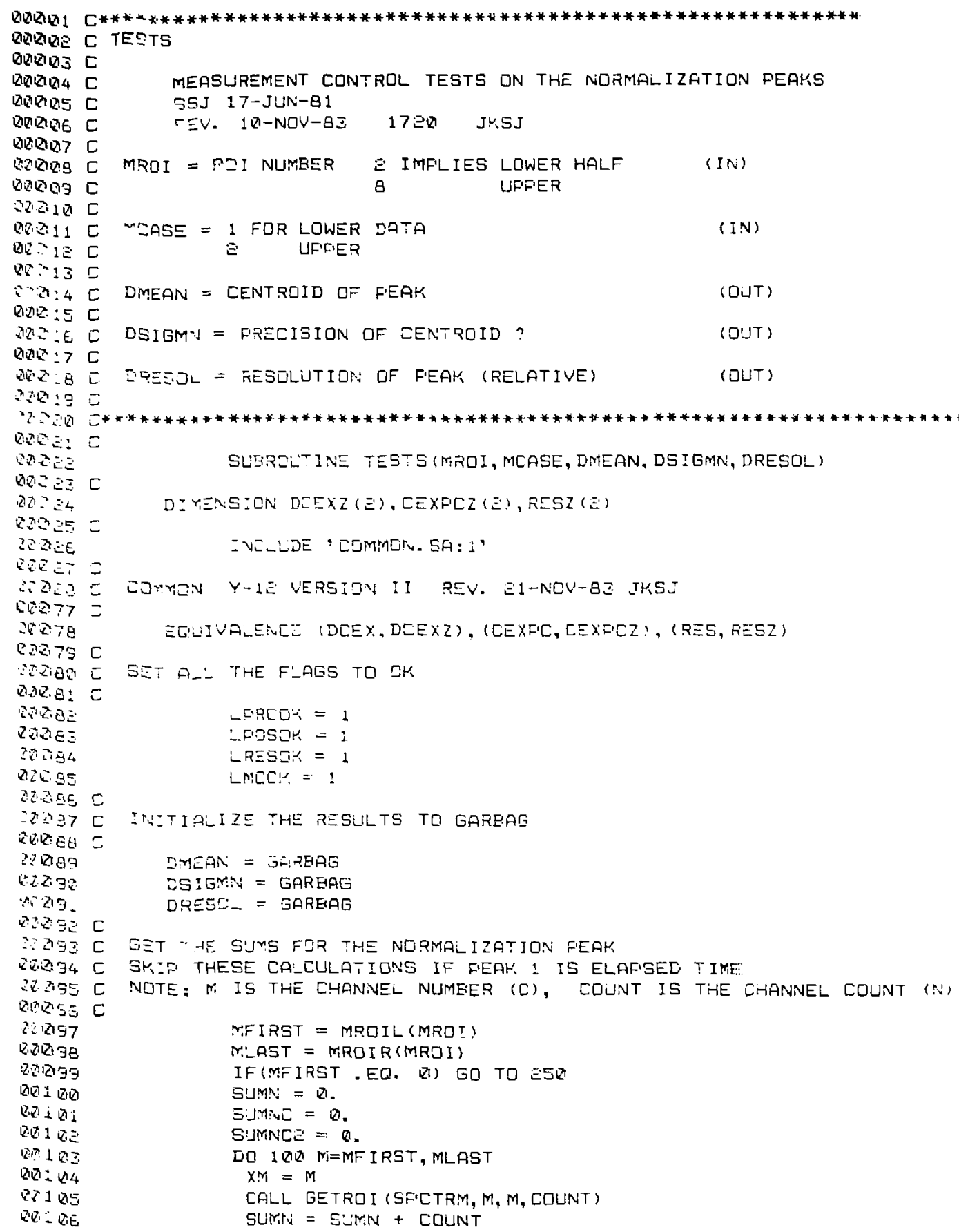




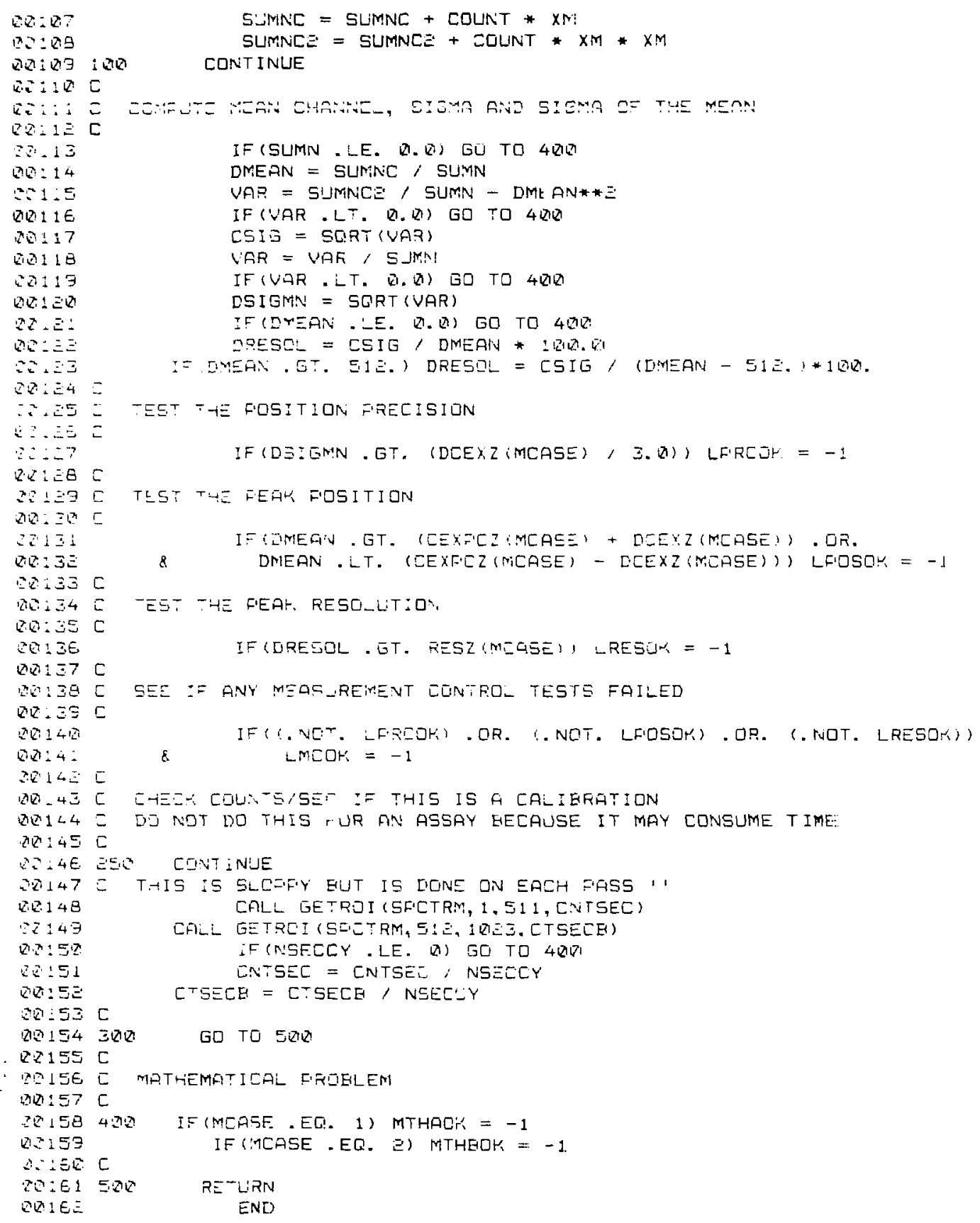




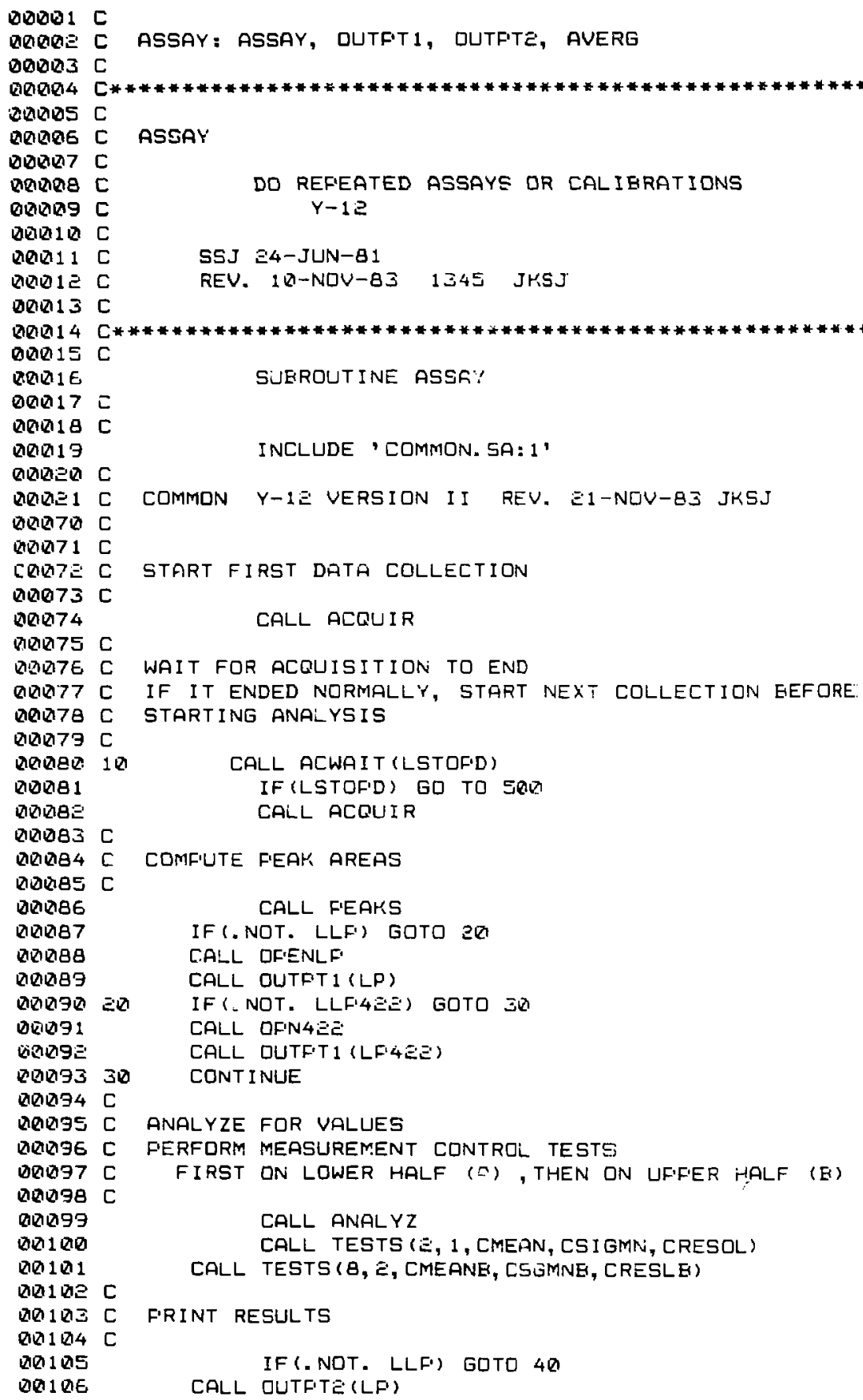




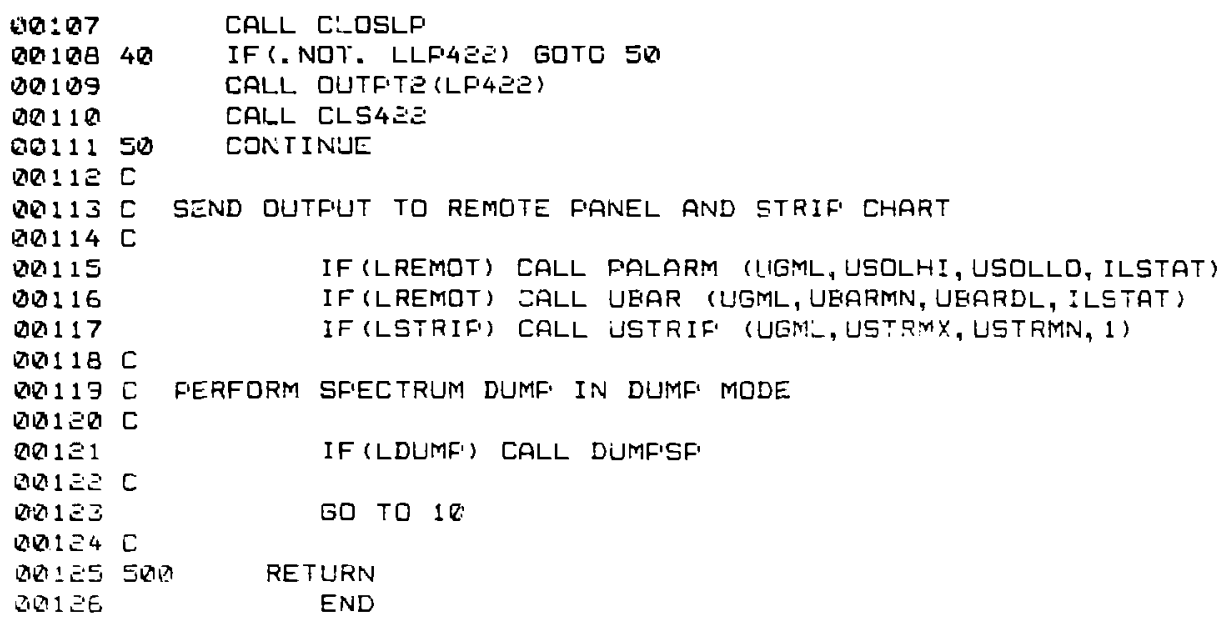




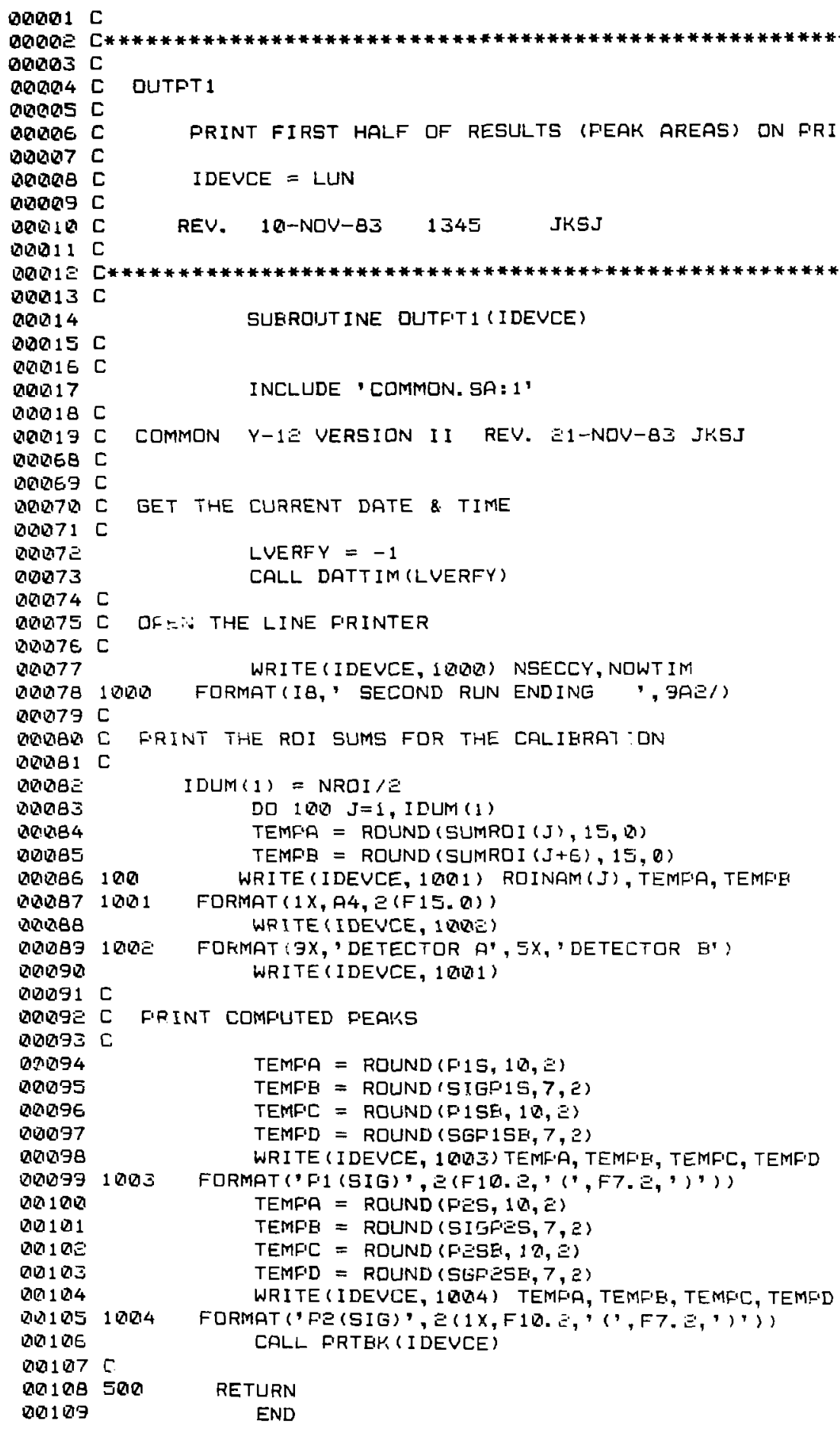




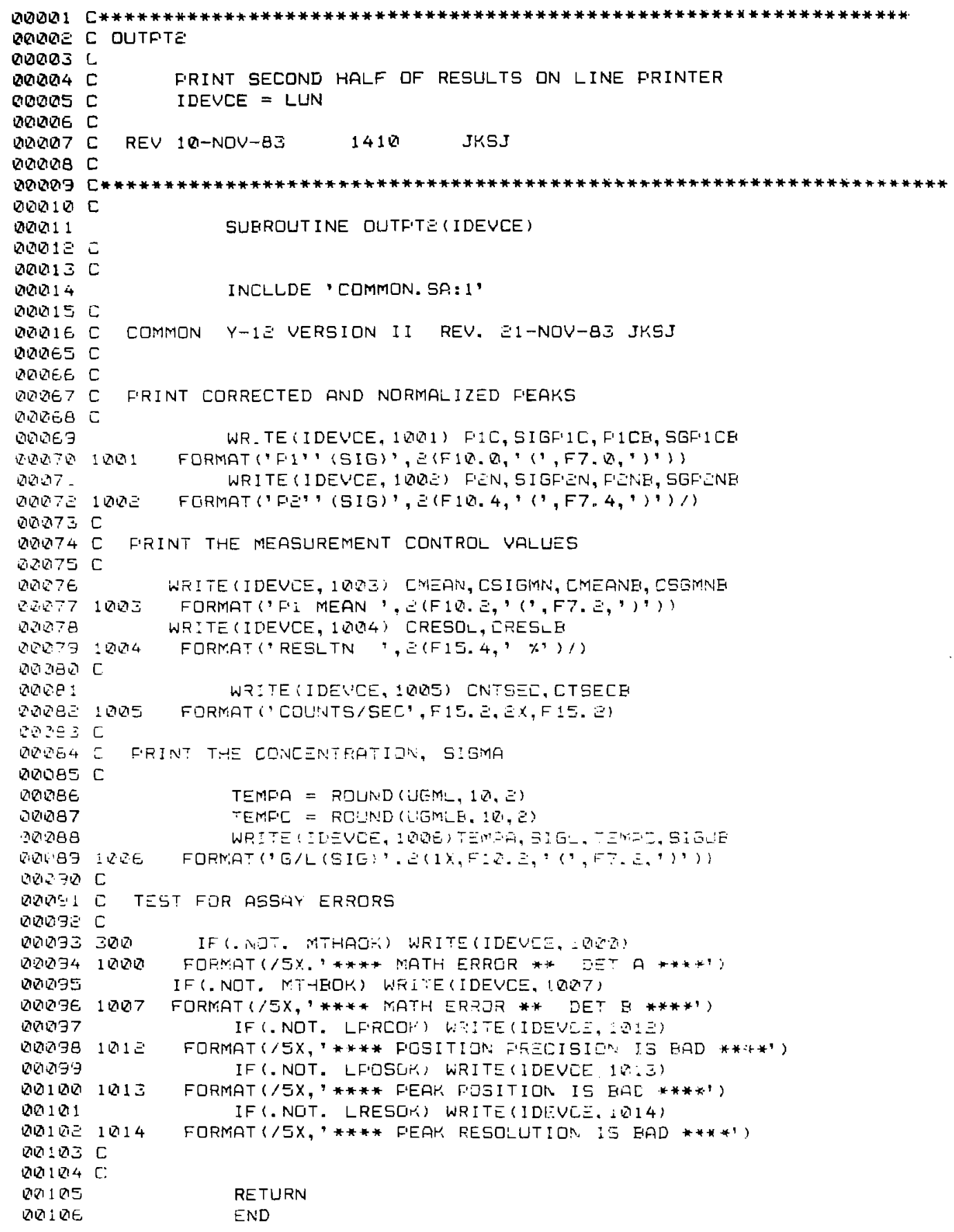

